\title{
Coherency and incoherency in neutrino-nucleus elastic and inelastic scattering
}

\author{
Vadim A. Bednyakov and Dmitry V. Naumov* \\ Joint Institute for Nuclear Research, Dubna, 141980 Moscow Region, Russia
}

(Received 22 June 2018; published 19 September 2018)

\begin{abstract}
Neutrino-nucleus scattering $\nu A \rightarrow \nu A$, in which the nucleus conserves its integrity, is considered. Our consideration follows a microscopic description of the nucleus as a bound state of its constituent nucleons described by a multiparticle wave function of a general form. We show that elastic interactions keeping the nucleus in the same quantum state lead to a quadratic enhancement of the corresponding cross section in terms of the number of nucleons. Meanwhile, the cross section of inelastic processes in which the quantum state of the nucleus is changed, essentially has a linear dependence on the number of nucleons. These two classes of processes are referred to as coherent and incoherent, respectively. Accounting for all possible initial and final internal states of the nucleus leads to a general conclusion independent of the nuclear model. The coherent and incoherent cross sections are driven by factors $\left|F_{p / n}\right|^{2}$ and $\left(1-\left|F_{p / n}\right|^{2}\right)$, where $\left|F_{p / n}\right|^{2}$ is a proton/neutron form factor of the nucleus, averaged over its initial states. Therefore, our assessment suggests a smooth transition between regimes of coherent and incoherent neutrino-nucleus scattering. In general, both regimes contribute to experimental observables. The coherent cross-section formula used in the literature is revised and corrections depending on kinematics are estimated. Consideration of only those matrix elements which correspond to the same initial and final spin states of the nucleus and accounting for a nonzero momentum of the target nucleon are two main sources of the corrections. As an illustration of the importance of the incoherent channel, we considered three experimental setups with different nuclei. As an example, for ${ }^{133} \mathrm{Cs}$ and neutrino energies of 30 $50 \mathrm{MeV}$, the incoherent cross section is about $10 \%-20 \%$ of the coherent contribution if the experimental detection threshold is accounted for. Experiments attempting to measure coherent neutrino scattering by solely detecting the recoiling nucleus, as is typical, might be including an incoherent background that is indistinguishable from the signal if the excitation gamma eludes its detection. However, as is shown here, the incoherent component can be measured directly by searching for photons released by the excited nuclei inherent to the incoherent channel. For a beam experiment these gammas should be correlated in time with the beam, and their higher energies make the corresponding signal easily detectable at a rate governed by the ratio of incoherent to coherent cross sections. The detection of signals due to the nuclear recoil and excitation $\gamma$ s provides a more sensitive instrument in studies of nuclear structure and possible signs of new physics.
\end{abstract}

DOI: $10.1103 /$ PhysRevD.98.053004

\section{INTRODUCTION}

The process of neutrino scattering, by means of $Z^{0}$-boson exchange, off a system of bonded particles provides a great laboratory to test principles of quantum physics and search for new phenomena. Under certain conditions the

\footnotetext{
* Corresponding author. dmitryvnaumov@gmail.com

Published by the American Physical Society under the terms of the Creative Commons Attribution 4.0 International license. Further distribution of this work must maintain attribution to the author(s) and the published article's title, journal citation, and DOI. Funded by SCOAP ${ }^{3}$.
}

corresponding interaction probability acquires an extra factor with respect to the case of scattering off free particles.

This extra factor, proportional to the number of scatterers, is a direct consequence of the principles of quantum physics. The probability of an outcome is determined by the absolute value squared of the sum of amplitudes corresponding to indistinguishable paths to realize this outcome. Neutrino-nucleus scattering in which the nucleus conserves its integrity is an example of this kind, as was observed by Freedman [1] more than four decades ago.

There are two distinct outcomes of such interactions: (i) the nucleus remains in the same quantum state and (ii) the state is changed. We refer to these cases as elastic and inelastic scatterings, respectively, because in (i) the 
energy transfer to the recoil nucleus is vanishingly small, while in (ii) it is apparently nonzero.

It was shown [2-5] that the cross section of elastic neutrino scattering off a nucleus is amplified with respect to a neutrino scattering off a single nucleon. The amplification factor for a spinless even-even nucleus reads

$$
\left|g_{V}^{n} N F_{n}(\boldsymbol{q})+g_{V}^{p} Z F_{p}(\boldsymbol{q})\right|^{2} \simeq N^{2}\left(g_{V}^{n}\right)^{2}\left|F_{n}(\boldsymbol{q})\right|^{2},
$$

where $Z$ and $N$ are the numbers of protons and neutrons, $g_{V}^{p / n}$ are proton/neutron couplings of the nucleon vector current, and $F_{p / n}(\boldsymbol{q})$ are proton/neutron form factors of the nucleus. The form factors approach unity if

$$
|\boldsymbol{q}| R_{A} \ll 1,
$$

where $R_{A}$ is the radius of the nucleus. The form factors vanish at $|\boldsymbol{q}| \rightarrow \infty$.

Neutrinos with energies below some tens of $\mathrm{MeV}$ predominately conserve the integrity of nucleons in neutrino-quark interactions with $Z^{0}$-boson exchange, allowing one to consider this process using an effective neutrinonucleon interaction in which the nucleon current is a sum of vector and axial currents.

The corresponding axial currents do not contribute significantly when a neutrino elastic scatters off of a spin-less nucleus due to the cancellation in the sum of amplitudes. The vector coupling $g_{V}^{p}=\frac{1}{2}-2 \sin ^{2} \theta_{W}$ of the proton is small $\left(g_{V}^{p} \approx 0.023\right)$ and is neglected in the approximate equality in Eq. (1). In our estimates, we used a best-fit value of $\sin ^{2} \theta_{W}=0.23865$, determined using low energy neutrino data and $\overline{\mathrm{MS}}$ renormalization scheme [6].

Freedman coined the terminology "coherent neutrinonucleus scattering" to emphasize the fact that the dependence of the corresponding cross section is quadratic in terms of the number of nucleons. This dependence was attributed to nearly identical amplitude phases corresponding to a neutrino scattering off nucleons.

His first calculations [1] were revised in a number of papers $[2-5,7-10]$. The impact of the nuclear structure models was studied in [11-15]. The importance of the coherent cross section was examined theoretically for a number of observables in astrophysics, like stellar collapse [16,17], supernovae [18-21], and in studies of physics beyond the standard model (SM) [3,22-31], electromagnetic properties of the neutrino [32], searches for sterile neutrinos $[33,34]$, and estimates of neutron density in the nucleus $[4,35]$. Coherent scattering of atomic systems was studied in [36,37], where the concept of neutrino optics was suggested for neutrinos with energies $\lesssim 10 \mathrm{keV}$.

Dating back to the seminal paper by Freedman [1], a number of experimental proposals [2,38-52] using reactor and accelerator neutrinos were suggested to observe neutrino-nucleus coherent scattering. This process is an unavoidable background in sensitive searches for dark matter [53-59]. The difficulty in observing coherent neutrino scattering lies in the detection of scattered nuclei with low kinetic energy of the order of some $\mathrm{keV}$ or tens of $\mathrm{keV}$.

The first experimental evidence for coherent neutrino-nucleus scattering was reported in 2017 by the COHERENT Collaboration [60-62], using CsI[Na] scintillator exposed to neutrinos with energies of tens of $\mathrm{MeV}$ produced by the Spallation Neutron Source (SNS) at the Oak Ridge National Laboratory [63-65].

Our motivation for this work was triggered by the following observation. At neutrino energies of some tens of $\mathrm{MeV}$, the three-momentum transfer $\boldsymbol{q}$ is large enough to break the condition in Eq. (2). For example, energy deposits observed in [63] correspond to $|\boldsymbol{q}| R_{A}$ sampling the interval $(1,2.7)$, and the elastic cross section should be suppressed. At higher energies, but still in the regime where the nucleus conserves its integrity, the elastic cross section vanishes and the neutrino-nucleus interaction probability must be determined by inelastic interactions. In general, the corresponding cross section should be given by a sum of elastic and inelastic cross sections, similar to the theory of the scattering of $x$ rays [66] and electrons [67] off an atom, and of slow neutrons off of matter constituents [68].

What should one expect about the "coherency" in inelastic processes? If this terminology is understood literally as the equality of phases of neutrino-nucleon scattering amplitudes, then one would conclude that inelastic processes should also be coherent, as in elastic processes, because there is no reason why these phases should be different. Should one then expect a quadratic dependence of the inelastic cross section in terms of the number of nucleons, similar to Eq. (1)? The corresponding literature, to best of our knowledge, lacks an appropriate theory for neutrino-nucleus interactions that could address these questions. This paper attempts to provide a theoretical framework accounting for elastic and inelastic neutrino-nucleus scattering of the process

$$
\nu A \rightarrow \nu A^{(*)},
$$

based on calculations from first principles. In Eq. (3), the possibility that the internal quantum state of a nucleus can be modified after an interaction is labeled by the $(*)$ superscript.

We show in this work that the cross section of the neutrino-nucleus elastic process is, indeed, quadratically dependent on the number of nucleons, while that for inelastic scattering exhibits a linear dependence. Elastic and inelastic cross sections also possess a distinct dependence on $\boldsymbol{q}$ : the former is driven by $\left|F_{p / n}\right|^{2}$, while the latter is governed by $1-\left|F_{p / n}\right|^{2}$. At the same time, the phases of corresponding neutrino-proton and neutrino-neutron amplitudes are all equal for protons and neutrons, respectively. This is at odds with the assumption that the difference of 
phases of the scattering amplitudes is responsible for loss of coherency $[17,32,69]$. Our arguments are discussed in what follows.

The paper is split into two parts. The first part is focused only on the main points of the derivation and discusses the results obtained. The second part, containing the necessary technical details, is organized in a set of Appendices.

In particular, a conceptual derivation of a general form of the cross section of the process in Eq. (3) is discussed in Sec. II. We review the paradigm of coherent scattering and suggest our concept in a simplified way in Sec. II A. The kinematics of elastic and inelastic scattering, and the corresponding amplitude and the cross section are discussed in Secs. II B and IIE, respectively. We refer to Appendices $\mathrm{A}-\mathrm{C}$ for full details of this derivation.

In Appendix A, we define the theoretical framework, reminding the reader of the decomposition of a quantum state in $x$ and $p$ bases for $n$-particle states, introducing notation and defining a general form of the wave function of the nucleus. In Appendix B, we compute the scattering amplitude and the cross section. In Appendix C, we summarize some details of our calculations of the scalar product of lepton and hadron currents, needed to calculate the scattering amplitude and the cross section.

In Sec. III, we discuss in detail the derived cross section. Coherent and incoherent regimes are discussed in Sec. III A. Our revision of the coherent cross section is discussed in Sec. III B. In Sec. IIIC, we discuss in some detail a proposal to detect transition $\gamma \mathrm{s}$ from excited nuclei inherent to incoherent processes. These $\gamma \mathrm{s}$ would provide both an additional background suppression and an independent observable sensitive to the form factor of the nucleus. In Appendix D, we provide an analogy with a mechanical system of two balls connected by a spring to illustrate the kinematics of coherent and incoherent scattering. The summary is drawn in Sec. IV.

The natural units $\hbar=c=1$ are used throughout the paper. Three-vectors are denoted by bold face. A fourvector $a$ has the following components: $a^{\mu}=\left(a^{0}, \boldsymbol{a}\right)$, enumerated by a greek index $\mu$. The Dirac spinors and $\gamma$-matrices are used in the Dirac basis and $\gamma_{5}=i \gamma_{0} \gamma_{1} \gamma_{2} \gamma_{3}$. The Feynman slash notation $\not k=\gamma^{\mu} a_{\mu}$ is used for a scalar product of a four-vector $a_{\mu}$ and Dirac $\gamma^{\mu}$-matrices. Quantum operators are denoted by the hat symbol, like $\hat{\boldsymbol{X}}$ for the position operator.

\section{ELASTIC AND INELASTIC NEUTRINO-NUCLEUS SCATTERING}

\section{A. Revising the paradigm}

We begin this section by reminding the reader of the paradigm of coherency in neutrino-nucleus scattering [17]. Two waves are considered coherent if they have the same frequencies, wave forms, and constant relative

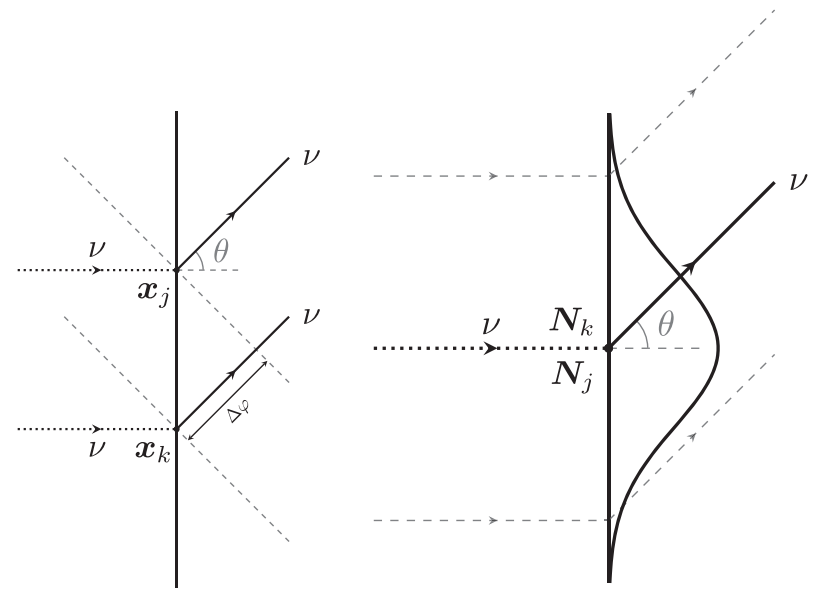

FIG. 1. Left panel: Front of incoming neutrino plane-wave (solid vertical line) scatters on nucleons at fixed positions, $\boldsymbol{x}_{j}$ and $\boldsymbol{x}_{k}$, respectively. Nonzero scattering angle $\theta$ develops the phase difference $\Delta \varphi=\boldsymbol{q}\left(\boldsymbol{x}_{j}-\boldsymbol{x}_{k}\right)$ of two fronts of scattered neutrino plane-waves (dashed lines) which leads to a loss of coherence. Right panel: Neutrino scatters off a $k$ th or $j$ th nucleon described by a wave function exemplified here as a Gaussian profile. The outgoing neutrino wave, as for any nucleon target, is a superposition of waves $e^{i \boldsymbol{q} \boldsymbol{x}_{k}}$ weighted by $\left|\psi_{n}\left(\boldsymbol{x}_{1} \ldots \boldsymbol{x}_{A}\right)\right|^{2}$.

phase. Coherence can lead to constructive and destructive interference.

A neutrino-nucleus interaction is a result of an individual neutrino scattering off of nucleons. Each such scattering off a $k$ th nucleon can be described by an amplitude $\mathcal{A}^{k}$.

If these nucleons are assumed to have definite coordinates $\boldsymbol{x}_{k}$, then, due to the translation invariance, $\mathcal{A}^{k}$ gets an additional factor $e^{i \boldsymbol{q} x_{k}}$ and the total amplitude reads

$$
\mathcal{A}=\sum_{k=1}^{A} \mathcal{A}^{k} e^{i \boldsymbol{q} x_{k}} .
$$

These individual amplitudes are coherent if, for any $k$, the phases $\boldsymbol{q} \boldsymbol{x}_{k}$ are nearly the same. This is fulfilled if the condition in Eq. (2) is satisfied.

The left panel of Fig. 1 depicts a neutrino scattering off of nucleons displaced from each other. The nonzero angle $\theta$ of the scattered neutrino leads to a loss of coherence.

Does this consideration of coherency remain appropriate when the assumption of the nucleon's definite position is released? In this case, the positions of nucleons are described by a multiparticle scalar wave function $\psi_{n / m}\left(\boldsymbol{x}_{1} \ldots \boldsymbol{x}_{A}\right)$, where the $n / m$ subscripts stand for the initial and final state of the nucleus. The amplitude in Eq. (4) could be generalized as

$$
\mathcal{A}_{n n}=\sum_{k=1}^{A} \mathcal{A}_{n n}^{k} f_{n n}^{k}(\boldsymbol{q}),
$$

where 


$$
\begin{aligned}
f_{m n}^{k}(\boldsymbol{q}) & =\left\langle m\left|e^{i \boldsymbol{q} \hat{\boldsymbol{X}}_{k}}\right| n\right\rangle \\
& =\int\left(\prod_{i=1}^{A} d \boldsymbol{x}_{i}\right) \psi_{m}^{*}\left(\boldsymbol{x}_{1} \ldots \boldsymbol{x}_{A}\right) \psi_{n}\left(\boldsymbol{x}_{1} \ldots \boldsymbol{x}_{A}\right) e^{i \boldsymbol{q} \boldsymbol{x}_{k}}
\end{aligned}
$$

is the transition matrix element of $e^{i q \hat{X}_{k}}$ with $\hat{\boldsymbol{X}}_{k}$ being the quantum position operator of the $k$ th nucleon.

In particular,

$$
f_{n n}^{k}(\boldsymbol{q})=\int\left(\prod_{i=1}^{A} d \boldsymbol{x}_{i}\right)\left|\psi_{n}\left(\boldsymbol{x}_{1} \ldots \boldsymbol{x}_{A}\right)\right|^{2} e^{i \boldsymbol{q} \boldsymbol{x}_{k}},
$$

defining the form factor of the nucleon bound in the nucleus, differs from the exponential factor $e^{i \boldsymbol{q} x_{k}}$ in two major respects: (i) $f_{n n}^{k}(\boldsymbol{q})$ does not depend on the coordinate of the $k$ th nucleon. All position variables are integrated out in Eq. (6). (ii) $f_{n n}^{k}(\boldsymbol{q})$ does not depend on the index $k$ (ignoring for simplicity a possible difference in form factors for protons and neutrons). This statement can be proven for both fermions and bosons by a change of integration variables, and accounting for symmetry properties of the wave function under interchange of its arguments.

Now, accounting for these properties of $f_{n n}^{k}(\boldsymbol{q})$, we conclude that phases of each individual amplitude in the total amplitude in Eq. (5) are all equal and the amplitudes are coherent for any $\boldsymbol{q}$, at variance with Eq. (4).

This conclusion does not mean to say that the total amplitude is not vanishing at large $\boldsymbol{q}$, because in this limit the form factor $f_{n n}(\boldsymbol{q})$ vanishes. What governs such dependence of $f_{n n}(\boldsymbol{q})$ ? Mathematically, the reason lies in the fast oscillation of the $e^{i q x_{k}}$ factor in the integral in Eq. (6), washing out the integrand function. The physical reason is in the incoherent summation of waves belonging to the wave function of a single nucleon extended over the size of the nucleus. Other physics arguments are discussed in Sec. II C and Appendix D.

One can argue that this conclusion seems to be in conflict with a wave function corresponding to the nucleons at fixed positions, assuming that

$$
\left|\psi_{n}\left(\boldsymbol{y}_{1} \ldots \boldsymbol{y}_{A}\right)\right|^{2} \propto \prod_{i} \delta^{3}\left(\boldsymbol{y}_{i}-\boldsymbol{x}_{i}\right),
$$

where $\boldsymbol{y}_{i}$ are variables and $\boldsymbol{x}_{i}$ are parameters. Then, Eq. (7) reduces to Eq. (4) in which every term has an individual phase in contrast to our statement. This antinomy appeared because of the assumption in Eq. (8) which breaks the principle of the particles identity. The latter requires that the multiparticle wave function should be either symmetric (bosons) or anti-symmetric (fermions) under exchange of its arguments. As a result it is not possible to state that the $i$ th particle has position $\boldsymbol{x}_{i}$ even if it is known that all particles occupy some fixed positions. Instead, the $i$ th particle can be at any point among the $x_{1} \ldots x_{A}$ fixed positions. Therefore, considering Eq. (7) with an appropriately symmetrized $\delta$-like wave function, one would identically obtain Eq. (4) for any index $k$ in agreement with our conclusion. Consideration of this antinomy is also helpful in understanding that the very form of Eq. (4) ignores the fundamental principle of quantum physics - the indistinguishability of particles.

The right panel of Fig. 1 displays a scattering picture accounting for a wave function of the nucleons exemplified here as a Gaussian profile. The summation of waves weighted by $\left|\psi_{n}\left(\boldsymbol{x}_{1} \ldots \boldsymbol{x}_{A}\right)\right|^{2}$ yields the scattered neutrino wave, as for any nucleon.

Therefore, according to our consideration, it is not appropriate to identify the diagonal terms in

$$
\begin{aligned}
\left|\mathcal{A}_{n n}\right|^{2} & =\left|f_{n n}(\boldsymbol{q})\right|^{2} \sum_{k, j} \mathcal{A}_{n n}^{k} \mathcal{A}_{n n}^{j *} \\
& =\left|f_{n n}(\boldsymbol{q})\right|^{2}\left(\sum_{k}\left|\mathcal{A}_{n n}^{k}\right|^{2}+\sum_{k \neq j} \mathcal{A}_{n n}^{k} \mathcal{A}_{n n}^{j *}\right)
\end{aligned}
$$

as due to incoherent interactions. Both diagonal and nondiagonal terms contribute equally to $\left|\mathcal{A}_{n n}\right|^{2}$, and with the same dependence on $\boldsymbol{q}$.

What, then, defines the incoherent interactions? Essentially, they are defined by processes in which the quantum state of the nucleus is changed $(n \neq m)$. Let us briefly highlight the main points of a derivation illustrating this statement, ignoring for a while complications due to spin, type of nucleon, possible dependence of $\mathcal{A}_{m n}^{k} \rightarrow \mathcal{A}_{0}$ on the indices, etc. (full details can be found in Appendix B).

Assume the nucleus is initially in the $n$th quantum state. If the experiment is not able to distinguish the final state of the nucleus, one should sum over all possible final states to get the observable proportional to

$$
|\mathcal{A}|^{2}=\sum_{m}\left|\mathcal{A}_{m n}\right|^{2}=\left|\mathcal{A}_{0}\right|^{2} \sum_{k, j} \sum_{m} f_{m n}^{k} f_{m n}^{j *}
$$

Using Eq. (6) one can rewrite Eq. (10) as

$$
\begin{aligned}
|\mathcal{A}|^{2} & =\left|\mathcal{A}_{0}\right|^{2} \sum_{k, j}\left\langle n\left|e^{-i q \hat{X}_{j}} \sum_{m}\right| m\right\rangle\left\langle m\left|e^{i q \hat{X}_{k}}\right| n\right\rangle \\
& =\left|\mathcal{A}_{0}\right|^{2} \sum_{k, j}\left\langle n\left|e^{-i q \hat{X}_{j}} e^{i q \hat{X}_{k}}\right| n\right\rangle,
\end{aligned}
$$

where we used the unity operator composed of nuclear states $\sum_{m}|m\rangle\langle m|=\hat{I}$.

One can define a two-particle real-valued correlation function

$$
G_{n n}(\boldsymbol{q}) \equiv G(\boldsymbol{q})=\left\langle n\left|e^{-i q \hat{X}_{j}} e^{i q \hat{X}_{k}}\right| n\right\rangle .
$$

If $k=j$, then $G(\boldsymbol{q})=1$. For $k \neq j, G(\boldsymbol{q})$ does not depend on values of $k, j$ as can be seen using the symmetry properties of the nucleus wave function. Combining Eqs. (6), (11) and (12) one gets 


$$
\begin{aligned}
|\mathcal{A}|^{2} & =\left|\mathcal{A}_{0}\right|^{2}(A+G(\boldsymbol{q}) A(A-1)) \\
& =\left|\mathcal{A}_{0}\right|^{2}\left(A^{2} G(\boldsymbol{q})+A(1-G(\boldsymbol{q}))\right),
\end{aligned}
$$

where $A$ gives the number of nucleons. The terms of $|\mathcal{A}|^{2}$ in Eq. (13), quadratically and linearly depending on $A$, are shaped by factors $G$ and $1-G$, respectively. These terms provide a smooth transition between coherent and incoherent regimes. One can observe that if the nucleus's multiparticle wave function is constructed as a product of single-particle wave functions, then $G(\boldsymbol{q})$ can be represented as $|F(\boldsymbol{q})|^{2}$, where $F(\boldsymbol{q})$ is the single-nucleon form factor of the nucleus.

The derivation of Eq. (13) does not indicate in a transparent way what the source of the quadratic and linearly dependent terms is. We conclude this section by showing that coherent and incoherent terms are due to processes in which the nucleus remains in the same quantum state or is changed, respectively. For this purpose, we rewrite Eq. (11) as

$$
\begin{aligned}
|\mathcal{A}|^{2}= & \left|\mathcal{A}_{0}\right|^{2} \sum_{k, j}\left\langle n\left|e^{-i q \hat{X}_{j}}\right| n\right\rangle\left\langle n\left|e^{i q \hat{X}_{k}}\right| n\right\rangle \\
& +\left|\mathcal{A}_{0}\right|^{2} \sum_{k, j} \sum_{m \neq n}\left\langle n\left|e^{-i q \hat{X}_{j}}\right| m\right\rangle\left\langle m\left|e^{i q \hat{X}_{k}}\right| n\right\rangle .
\end{aligned}
$$

The first line gives immediately

$$
\left|\mathcal{A}_{0}\right|^{2} A^{2}|F(\boldsymbol{q})|^{2},
$$

which could be identified as a coherent term in Eq. (13). The second line can be presented as

$$
\left|\mathcal{A}_{0}\right|^{2}\left(A\left(1-|F(\boldsymbol{q})|^{2}\right)+\sum_{k \neq j} \operatorname{cov}\left(e^{-i \boldsymbol{q} \hat{X}_{j}}, e^{i q \hat{\boldsymbol{X}}_{k}}\right)\right),
$$

where the covariance of quantum operators reads

$$
\begin{aligned}
\operatorname{cov}\left(e^{-i q \hat{X}_{j}}, e^{i q \hat{X}_{k}}\right)= & \left\langle n\left|e^{-i q \hat{X}_{j}} e^{i q \hat{X}_{k}}\right| n\right\rangle \\
& -\left\langle n\left|e^{-i q \hat{X}_{j}}\right| n\right\rangle\left\langle n\left|e^{i q \hat{X}_{k}}\right| n\right\rangle .
\end{aligned}
$$

The covariance terms are identically zero for a multiparticle wave function constructed as a product of single-particle wave functions and the second line of Eq. (14) reads

$$
\left|\mathcal{A}_{0}\right|^{2} A\left(1-|F(\boldsymbol{q})|^{2}\right) \text {. }
$$

Therefore, one can conclude that an elastic process [first line in Eq. (14)] yields the coherent term in Eq. (15), while inelastic processes all together [second line in Eq. (14)] yield the incoherent term in Eq. (18).

One can find a certain analogy with the theory of neutrino oscillations in which the integration over an unobserved time of neutrino emission leads to an incoherent
$L$-independent term in the oscillation probability formula (see, e.g., in [70,71]).

Attribution of elastic and inelastic processes as contributing to the coherent and incoherent interactions was also done in $[20,72-74]$ where the authors performed numerical calculations of the corresponding cross sections within appropriate nuclear models.

\section{B. Kinematics of elastic and inelastic neutrino-nucleus scattering}

In general, one should consider the treatment of neutrinonucleus interactions using wave packets. The corresponding formalism was developed (see, e.g., Ref. [70,75]) and some potentially interesting effects for elastic neutrino-nucleus scattering could be envisaged and examined. We simplify our treatment by considering the initial and final states as having definite momenta.

Let us denote by $k=\left(E_{\nu}, \boldsymbol{k}\right)$ and $k^{\prime}=\left(E_{\nu}^{\prime}, \boldsymbol{k}^{\prime}\right)$ the fourmomenta of incoming and outgoing neutrino, and by $P_{n}$ and $P_{m}^{\prime}$ the four-momenta of initial and final state nuclei, respectively.

The total energy $P_{n}^{0}$ of a nucleus state $\left|P_{n}\right\rangle$ reads as $E_{\boldsymbol{P}}+\varepsilon_{n}$, where $\varepsilon_{n}$ is an internal energy of the nucleus state. In the laboratory frame, energy $E_{\nu}^{\prime}$ of the outgoing neutrino depends on angle $\theta$ between $\boldsymbol{k}$ and $\boldsymbol{k}^{\prime}$

$$
E_{\nu}^{\prime}=\frac{m_{A}\left(E_{\nu}-\Delta \varepsilon_{m n}\right)-E_{\nu} \Delta \varepsilon_{m n}+\Delta \varepsilon_{m n}^{2} / 2}{m_{A}+E_{\nu}(1-\cos \theta)-\Delta \varepsilon_{m n}},
$$

where

$$
\Delta \varepsilon_{m n}=\varepsilon_{m}-\varepsilon_{n}
$$

is the difference of energies of the $|m\rangle$ and $|n\rangle$ states. Absolute values of the four-momentum transfer vector, $q=\left(q_{0}, \boldsymbol{q}\right)$, read

$$
\begin{aligned}
q_{0} & =E_{\nu}-E_{\nu}^{\prime}=\Delta \varepsilon_{m n}+T_{A}, \\
|\boldsymbol{q}| & =\left(E_{\nu}^{2}+E_{\nu}^{\prime 2}-2 E_{\nu} E_{\nu}^{\prime} \cos \theta\right)^{1 / 2} \simeq\left(2 m_{A} T_{A}\right)^{1 / 2},
\end{aligned}
$$

where $T_{A}$ is the kinetic energy of the scattered nucleus, calculated below.

In the neutrino-nucleus center-of-mass frame, $q^{2}$ reads

$$
q^{2}=-4 E_{\nu, n}^{\star} E_{\nu, m}^{\star} \sin ^{2} \frac{\theta_{\star}}{2}
$$

where

$$
E_{\nu, n}^{\star}=\frac{s_{A, n}-m_{A, n}^{2}}{2 \sqrt{s_{A}}}
$$

is the energy of neutrino scattering off a nucleon state $|n\rangle$, $s_{A, n}=\left(k+P_{n}\right)^{2}$ and $m_{A, n}=m_{A}+\varepsilon_{n}$. 
Minimum and maximum values of $q^{2}$ correspond to $\sin ^{2} \frac{\theta_{\star}}{2}=1$ and 0 , respectively,

$$
\begin{aligned}
& q_{\min }^{2}=-4 E_{\nu, n}^{\star} E_{\nu, m}^{\star}=\frac{-4 E_{\nu}^{2}}{\sqrt{\left(1+\frac{2 E_{\nu}}{m_{A, n}}\right)\left(1+\frac{2 E_{\nu}}{m_{A, m}}\right)}}, \\
& q_{\max }^{2}=0 .
\end{aligned}
$$

For heavy nuclei with $\Delta \varepsilon_{m n}$ of the order of hundreds keV and experimentally detectable signals produced by a release of kinetic energy of the scattered nucleus, $q^{2}$ can be approximated as

$$
q^{2} \approx-q^{2} \simeq-2 m_{A} T_{A} .
$$

Assuming the initial nucleus is at rest, the kinetic energy of its recoil reads

$$
T_{A}=\sqrt{m_{A}^{2}+\boldsymbol{q}^{2}}-m_{A} .
$$

Using Eq. (19) and assuming $m_{A} \gg E_{\nu}$, the kinetic energy $T_{A}$ of the scattered nucleus becomes

$$
T_{A} \approx \frac{E_{\nu}\left(E_{\nu}-\Delta \varepsilon_{m n}\right)(1-\cos \theta)+\Delta \varepsilon_{m n}^{2} / 2}{m_{A}} .
$$

Here we will examine the kinetic energy for a few cases of interest. (i) Forward scattering of the neutrino corresponds to $\cos \theta=1$ and yields the minimal kinetic energy of the nucleus

$$
T_{A}^{\min }=\lim _{\cos \theta \rightarrow 1} T_{A} \approx \frac{\Delta \varepsilon_{m n}^{2} / 2}{m_{A}},
$$

which is zero for $m=n$ because no energy, nor threemomentum, is transferred in this case. For $m \neq n$, the energy $q_{0}=\Delta \varepsilon_{m n}$ is transferred to the nucleus as well as the three-momentum $\boldsymbol{q}$, equal in magnitude to $q_{0}$ for forward scattering, thus yielding $T_{A}=\boldsymbol{q}^{2} / 2 m_{A}$.

(ii) Backward scattering corresponds to $\cos \theta=-1$ and yields the maximal kinetic energy of the nucleus

$$
T_{A}^{\max }=\lim _{\cos \theta \rightarrow-1} T_{A} \approx \frac{\left(2 E_{\nu}-\Delta \varepsilon_{m n}\right)^{2}}{2 m_{A}},
$$

which can be understood as follows. For $m=n$, no energy is transferred to the nuclear structure, while the transferred three-momentum is equal to double the initial neutrino energy (backward scattering). Thus, $T_{A}=\left(2 E_{\nu}\right)^{2} / 2 m_{A}$. For $m \neq n$, the energy $\Delta \varepsilon_{m n}$ transferred to the nucleus must be subtracted from the total transfered three-momentum $2 E_{\nu}$, thus leading to Eq. (29).

(iii) In general, the kinetic energy of the scattered nucleus is smaller if the nucleus changes its quantum state
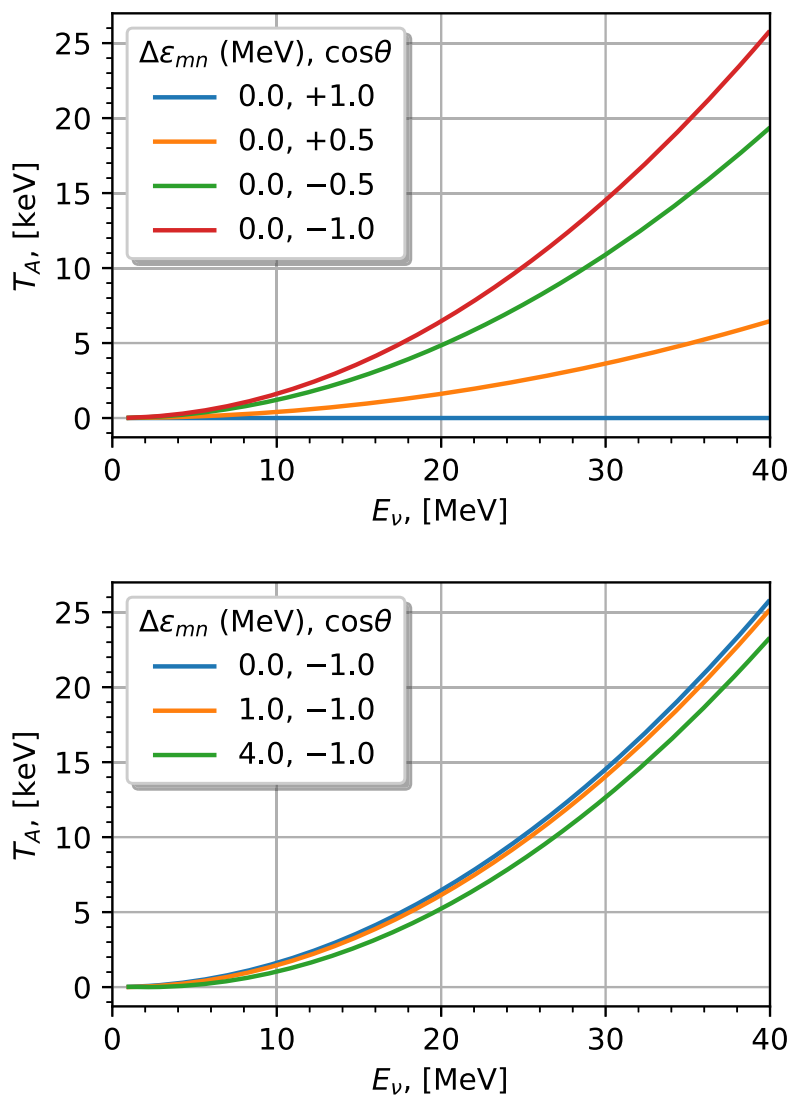

FIG. 2. Expected kinetic energy of nucleus ${ }^{133} \mathrm{Cs}$ scattered in an elastic interaction with a neutrino as a function of its energy. The upper plot corresponds to $\Delta \varepsilon_{m n}=0$ and four different values of $\cos \theta$, where $\theta$ is the neutrino scattering angle. The lower plot illustrates the impact of nonzero $\Delta \varepsilon_{m n}$ for a fixed value $\cos \theta=-1$.

$(m \neq n)$ with respect to the case when $m=n$. Effectively, this can be described by a decrease of neutrino energy by $\Delta \varepsilon_{m n}$, which could be significant when $E_{\nu}$ and $\Delta \varepsilon_{m n}$ are comparable.

For heavy nuclei, like ${ }^{133} \mathrm{Cs}$ or ${ }^{127} \mathrm{I}$, used by the COHERENT experiment [63], the first excitation energies are of the order of $\simeq 100 \mathrm{keV}$, which are small corrections compared to the tens-of-MeV neutrino energies produced by the Spallation Neutron Source. Therefore, the kinetic energy of the recoil nucleus is of the same order of magnitude for both elastic and inelastic scatterings.

In Fig. 2, we show the expected kinetic energy of the recoil nucleus ${ }^{133} \mathrm{Cs}$ as a function of its kinetic energy, illustrating the impact of $\Delta \varepsilon_{m n}$ and $\cos \theta$. A strong dependence on neutrino scattering angle $\theta$ is evident from the upper panel of Fig. 2. The effect of nonzero values of $\Delta \varepsilon_{m n}$, displayed in the bottom panel of Fig. 2, is also present, but it is significantly smaller than the angular dependence. The reason for the weaker dependence due to nonzero values of $\Delta \varepsilon_{m n}$ is in the partial compensation due to the $\Delta \varepsilon_{m n}^{2} / 2$ term in the numerator of Eq. (27) at $E_{\nu} \simeq \Delta \varepsilon_{m n}$, and irrelevance of $\Delta \varepsilon_{m n}$ when $E_{\nu} \gg \Delta \varepsilon_{m n}$. 


\section{Kinematic paradox}

As we show in what follows, the coherent enhancement of the interaction probability corresponds to neutrinonucleus scattering in which the nucleus remains in the same quantum state. This intuitively evident statement might seem to result in the following kinematic paradox. Both the nucleon and the nucleus acquire the same threemomentum $\boldsymbol{q}$. Assuming both of them are initially at rest, one arrives at the kinetic energy $T_{N}=\boldsymbol{q}^{2} / 2 m_{N}$ of the nucleon right after an interaction, which is a factor $m_{A} / m_{N}$ larger than the kinetic energy $T_{A}=\boldsymbol{q}^{2} / 2 m_{A}$ of the nucleus. Since the nucleus remains in the same quantum state with the same internal energy, $T_{N}$ and $T_{A}$ must be equal to each other, and to the difference $E_{\nu}-E_{\nu}^{\prime}$.

This paradox appears because some of the assumptions are incorrect. In particular, while the assumption that the nucleon is at rest seems to be quite reasonable given that the average nucleon momentum, $\boldsymbol{p}$, is much smaller than its mass, $m_{N}$, this assumption leads to the paradox.

Let us require that $T_{N}$ and $T_{A}$ are equal to each other. This requirement cannot be satisfied for any nucleon momentum $\boldsymbol{p}$. One can find a compatible $\boldsymbol{p}$ using energy conservation

$$
\frac{(\boldsymbol{p}+\boldsymbol{q})^{2}}{2 m_{N}}-\frac{\boldsymbol{p}^{2}}{2 m_{N}}=\frac{\boldsymbol{q}^{2}}{2 m_{A}} .
$$

Searching for a solution where $\boldsymbol{p}$ is proportional to $\boldsymbol{q}$, $\boldsymbol{p}=\alpha \boldsymbol{q}$, one finds the nucleon momentum to be

$$
\boldsymbol{p}=-\frac{\boldsymbol{q}}{2}\left(1-\frac{m_{N}}{m_{A}}\right) \text {. }
$$

Therefore, energy-momentum conservation and the requirement that the nucleus does not change its state after an interaction provides a qualitative picture of the coherent neutrino-nucleus scattering process, displayed symbolically in Fig. 3. Here we discuss a few features of this interesting observation. (i) Not every nucleon in the nucleus can interact with a neutrino in such a way that after the interaction the nucleus remains in the same state. Only those nucleons which happen to have a momentum compatible with Eq. (31) are appropriate targets.

(ii) The wave function of the nucleons provides us a distribution of the nucleon's momenta. Large nucleon momenta are, in general, less probable than smaller momenta. This explains qualitatively why at large $\boldsymbol{q}$ the enhancement factor in Eq. (1) vanishes, contrary to the case of small $\boldsymbol{q}$ for which the chance to find a nucleon with an appropriate momentum is relatively large. Mathematically, this suppression is given by $|F(\boldsymbol{q})|^{2}$.

This consideration could be extended to the case of incoherent neutrino-nucleus scattering, when the nucleus changes its intrinsic quantum state $|n\rangle \rightarrow|m\rangle$ and $n \neq m$. Equation (30) must be generalized to account for nonzero differences of energy levels $\Delta \varepsilon_{m n}$

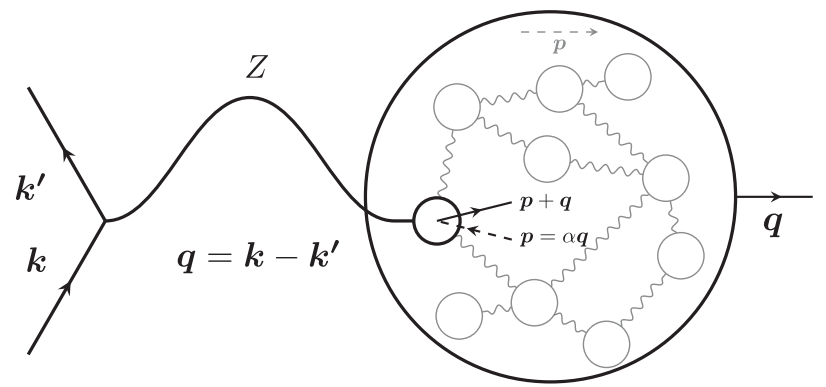

FIG. 3. A qualitative picture of a coherent neutrino-nucleus interaction. A neutrino interacts with a nucleon initially having a particular momentum $\boldsymbol{p}=\alpha \boldsymbol{q}$ aligned along $\boldsymbol{q}$ and given by Eq. (31). Since the nucleus initially is at rest, all the nucleons except the target one have a momentum $-\boldsymbol{p}$ shown by line dashed. The final momentum $\boldsymbol{p}+\boldsymbol{q}=(1+\alpha) \boldsymbol{q}$ of the target nucleon is also aligned along $\boldsymbol{q}$. In the figure, an angle between the $\boldsymbol{p}$ and $\boldsymbol{p}+\boldsymbol{q}$ vectors differs from $\pi$ for visual clarity. After the interaction the increased energy of the target nucleon and acquired three-momentum $\boldsymbol{q}$ are transferred to the entire nucleus, leaving the internal quantum state of the latter unchanged. A Z -boson having a wavelength comparable to the size of the nucleus produces a coherent enhancement of scattering amplitudes.

$$
\sqrt{E_{\boldsymbol{p}}^{2}+2 \boldsymbol{p} \boldsymbol{q}+\boldsymbol{q}^{2}}-E_{\boldsymbol{p}}-\frac{\boldsymbol{q}^{2}}{2 m_{A}}=\Delta \varepsilon,
$$

where $E_{\boldsymbol{p}}=\sqrt{m_{N}^{2}+\boldsymbol{p}^{2}}$. Equation (32) does not use a nonrelativistic approximation because for small values of $\boldsymbol{q}$, its solution $\boldsymbol{p}$ can be comparable to the nucleon mass.

Splitting $\boldsymbol{p}$ into a sum of components: longitudinal $\boldsymbol{p}_{L}$ and transverse $\boldsymbol{p}_{T}$ to $\boldsymbol{q}$, one can find an exact solution of Eq. (32)

$$
p_{L}=-\frac{|\mathbf{q}|}{2}\left(1-\sqrt{\beta} \sqrt{1+\frac{4 m_{N, T}^{2}}{\mathbf{q}^{2}(1-\beta)}}\right),
$$

where

$\beta=\frac{E_{m n}^{2}}{\mathbf{q}^{2}}, \quad E_{m n}=\frac{\mathbf{q}^{2}}{2 m_{A}}+\Delta \varepsilon_{m n}, \quad m_{N, T}^{2}=m_{N}^{2}+p_{T}^{2}$.

In Fig. 4, we display the solution in Eq. (33) as a function of $|\boldsymbol{q}|$. For $\Delta \varepsilon_{m n}=0$, the solution in Fig. 4 reproduces that in Eq. (31). In this case, $\beta$ from Eq. (34) is vanishingly small

$$
\beta=\frac{\boldsymbol{q}^{2}}{4 m_{A}^{2}}=\frac{T_{A}}{2 m_{A}} \ll 1
$$

and

$$
p_{L} \simeq-\frac{\left|\boldsymbol{q}_{L}\right|}{2}\left(1-\frac{m_{N, T}}{m_{A}}\right)
$$

coinciding with Eq. (31) for $p_{T}=0$. One can observe that the longitudinal momentum of the nucleon in coherent neutrino-nucleus scattering is always aligned opposite to 


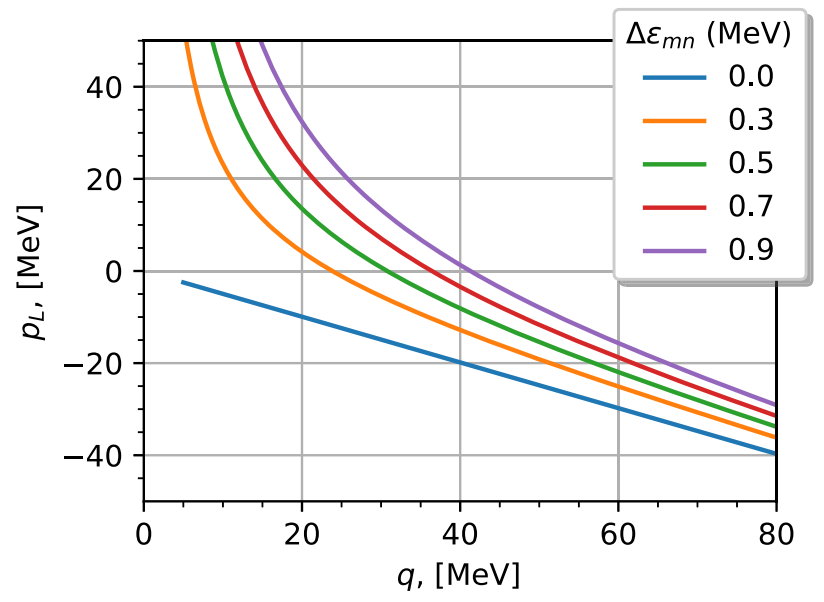

FIG. 4. Longitudinal component $p_{L}$ of the nucleon momentum corresponding to the energy-momentum conservation in neutrino-nucleus scattering.

the transfered three-momentum $\boldsymbol{q}$. For $\Delta \varepsilon_{m n} \neq 0$, the solution of Eq. (32) is drastically different for small $\boldsymbol{q}$. Here we analyze different regions of three-momentum transfer: (i) At $\boldsymbol{q}=0$, Eq. (32) has no solution, which simply means that at zero energy-momentum transfer an excitation of the nucleus is impossible.

(ii) At smallest $|\boldsymbol{q}|$ approaching its minimum possible value $\left|\boldsymbol{q}_{\min }\right|=\Delta \varepsilon_{m n}+\frac{\Delta \varepsilon_{m n}^{2}}{2 m_{A}}$, the solution of Eq. (33) diverges, $p_{L} \rightarrow \infty$, whence a nonrelativistic approximation in Eq. (32) is not appropriate at small $\boldsymbol{q}$. The chance to find a nucleon in the nucleus with such a momentum is vanishingly small. Therefore, for small $|\boldsymbol{q}|$ the incoherent scattering is significantly suppressed, in opposition to the coherent interaction.

(iii) With increasing $|\boldsymbol{q}|$ there is a good chance to find a transition $|n\rangle \rightarrow|m\rangle$ with $\Delta \varepsilon_{m n}$ yielding $\boldsymbol{p} \approx 0$ for which the suppression is minimal. Again, this dependence is exactly opposite to the coherent scattering.

(iv) These kinematic considerations give a qualitative understanding, yet do not provide a complete picture of the dependence of the neutrino-nucleus scattering upon $\boldsymbol{q}$. In $|n\rangle \rightarrow|m\rangle(n \neq m)$ transitions, the matrix element $\left\langle m\left|e^{i q \hat{\boldsymbol{X}}}\right| n\right\rangle$, where $\hat{\boldsymbol{X}}$ is the position operator, determines the actual functional dependence. A quantitative mathematical framework is developed in Appendix B 1.

As a useful and simple illustration of transitions for which the internal state is changed or unchanged, in Appendix D we consider a mechanical analogy of a system of two balls with equal masses $m$ connected by a massless spring having nonzero rigidity.

\section{Scattering amplitude}

Our calculation follows a microscopic description of neutrino-nucleus scattering as a result of the neutrinonucleon interaction.
We consider a Fock state $\left|P_{n}\right\rangle$ of a nucleus with fourmomentum $P_{n}$ being in the $n$-th quantum state as a superposition of free nucleons states weighted with their bound state wave function. The latter is explicitly factorized into a product of the wave functions describing the internal structure of the nucleus and motion of their center-of-mass. The internal wave function depends on $A-1$ threemomenta because one three-momentum variable is used to describe the motion of the nucleus.

It is convenient to refer to the Fock state $|n\rangle$, describing the nucleus in the $n$-th quantum state at rest. At zero nucleus momentum, both $\left|P_{n}\right\rangle$ and $|n\rangle$ states describe the same quantum state but still differ by their normalizations given in Eqs. (A22) and (A23). The details of this consideration are summarized in Appendix A.

A priori, one does not know the initial, $|n\rangle$, and final, $|m\rangle$, internal states of the nucleus. Therefore, all possible transitions must be considered. The matrix element $i \mathcal{M}_{m n}$, corresponding to the process in Eq. (3) keeping only the leading order terms of Fermi constant $G_{F}$, reads

$i \mathcal{M}_{m n}=i \frac{G_{F}}{\sqrt{2}} \frac{m_{A}}{m_{N}} C_{1, m n}^{1 / 2} \sum_{k=1}^{A} \sum_{s r} f_{m n}^{k} \lambda^{m n}(s, r)\left(l, h_{s r}^{k}\right)$,

where $m_{N}$ and $m_{A}$ are masses of the nucleon and nucleus, respectively, and $C_{m n, 1}$ is a function of the order of unity defined in Eq. (B19). Details of the derivation can be found in Appendix B 1.

Functions $f_{m n}^{k}(\boldsymbol{q}) \equiv\left\langle m\left|e^{i \boldsymbol{q} \hat{\boldsymbol{X}}_{k}}\right| n\right\rangle$, where $\hat{\boldsymbol{X}}_{k}$ stands for the position operator of the $k$ th nucleon, are transition form factors for $m \neq n$ and $n$-state form factors for $m=n$, defined in Eq. (B14).

$\left(l, h_{s r}^{k}\right)$ is the scalar product of the lepton $(l)$ and $k$ th nucleon's $\left(h_{s r}^{k}\right)$ neutral weak currents. For their definition, refer to Eqs. (B4) and (B25), respectively.

$\lambda^{m n}(s, r)$ is a spin transition amplitude between the $|n\rangle$ and $|m\rangle$ states of the nucleus. It depends on initial, $r$, and final, $s$, doubled spin projection on the given axis of the scattered nucleon. For a definition, refer to Eqs. (B11), (B12), and (B17). The amplitude in Eq. (37) is a sum of neutrino-nucleon amplitudes, each proportional to the scalar product of the lepton and nucleon currents, weighted by two factors, each not exceeding unity.

Given the definition of $f_{m n}^{k}$ in Eq. (B14) and the symmetry properties of the nucleus wave function, one can conclude that $f_{m n}^{k}$ does not depend on the number $k$, but only on the type of nucleon $k$ points to.

Therefore, all amplitudes in Eq. (37) have the same phase and thus are "coherent" in the literal sense of this terminology.

One can see that $f_{m n}^{k}(\boldsymbol{q})=\left\langle m\left|e^{i \boldsymbol{q} \hat{\boldsymbol{X}}_{k}}\right| n\right\rangle$ is a generalization of the $e^{i q x_{k}}$ quantum-mechanical factor used by Freedman in [1]. Section II A can be referred for a discussion of an important difference between these two factors. 
$f_{m n}^{k}$ is most important in understanding the mechanisms of the quadratic and linear dependence of the observable cross section on the number of nucleons.

Let us examine the form factor $f_{m n}^{k}$ for elastic and inelastic scatterings.

(i) In the case of elastic scattering,

$$
\lim _{q \rightarrow 0}\left\langle n\left|e^{i q \hat{X}_{k}}\right| n\right\rangle \rightarrow 1,
$$

and one expects a quadratic dependence of the cross section on the number of nucleons. For $\boldsymbol{q} \rightarrow \infty$, the matrix element vanishes

$$
\lim _{q \rightarrow \infty}\left\langle n\left|e^{i q \hat{X}_{k}}\right| n\right\rangle \rightarrow 0
$$

and the elastic cross section must also vanish. Therefore, the elastic scattering has the properties of a "coherent" process in the terminology of Freedman.

(ii) In the case of inelastic scattering,

$$
\lim _{q \rightarrow 0}\left\langle m\left|e^{i q \hat{\boldsymbol{X}}_{k}}\right| n\right\rangle \rightarrow 0,
$$

according to the normalization $\langle m \mid n\rangle=0$ for $n \neq m$ [see Eq. (A23)]. For a nonzero $\boldsymbol{q}$, the matrix element $\left\langle m\left|e^{i q \hat{X}_{k}}\right| n\right\rangle \neq 0$ in general and, as we show in Sec. II E, the cross section is a linear function of the number of nucleons once all possible initial and final states are accounted for. Since this result could be obtained by summing up the absolute values of the amplitudes squared one can refer to this case as incoherent scattering.

\section{E. Cross section}

The corresponding differential cross section reads

$$
\frac{d \sigma_{m n}}{d T_{A}}=\frac{\left|i \mathcal{M}_{m n}\right|^{2}}{2^{5} \pi E_{\nu}^{2} m_{A}} C_{2, m n},
$$

where $C_{2, m n}$ is a function of the order of unity given in Eq. (B23). As we show in App. C [see Eq. (C33)] the matrix-element squared, $\left|i \mathcal{M}_{m n}\right|^{2}$, is independent of the azimuthal angle $\varphi$, therefore we integrated over this variable in Eq. (41).

An observable cross section can be obtained by averaging over all possible initial states $|n\rangle$ and summing up over all possible final states $|m\rangle$

$$
\frac{d \sigma}{d T_{A}}=\sum_{n, m} \omega_{n} \frac{d \sigma_{m n}}{d T_{A}}
$$

where $\omega_{n}$ is a statistical weight to find an initial nucleus in a quantum state $|n\rangle$ at given ambient temperature.
In what follows, we do not need an explicit form of $\omega_{n}$ normalized as

$$
\sum_{n} \omega_{n}=1
$$

The matrix-element squared, $\left|i \mathcal{M}_{m n}\right|^{2}$, has inside it a summation $\sum_{k, j}$ over two indexes enumerating the scattered nucleons.

In Appendix B 2, it is shown that terms in Eq. (42), corresponding to elastic neutrino-nucleus scattering $\left(\sum_{n=m}\right)$, keep both indexes, $k$ and $j$, giving rise to a quadratic dependence of the cross section as a function of the number of nucleons. In contrast, terms in Eq. (42), corresponding to inelastic neutrino-nucleus scattering $\left(\sum_{n \neq m}\right)$, are to a good accuracy proportional to $\delta_{k j}$, which automatically yields a linear dependence on the cross section as a function of the number of nucleons.

Therefore, the observable cross section can be written as

$$
\begin{aligned}
\frac{d \sigma}{d T_{A}}= & \frac{4 G_{F}^{2} m_{A}}{\pi}\left(g_{i} \sum_{f=n, p} \sum_{k=1}^{A_{f}} \sum_{s, r}\left|\lambda_{s r}^{f}\right|^{2}\left|\left(l, h_{s r}^{f}\right)\right|^{2}\left(1-\left|F_{f}\right|^{2}\right)\right. \\
& \left.+g_{c}\left|\sum_{f=n, p} \sum_{k=1}^{A_{f}} \sum_{r}\left(l, h_{r r}^{f}\right) F_{f}\right|^{2}\right),
\end{aligned}
$$

where $\left|\lambda_{s r}^{p / n}\right|^{2}$ and $g_{i / c}$ are determined factorizing, respectively $\left|\lambda_{s r}^{m n}\right|^{2}$ given by Eq. (B18) and $g^{m n}$ defined by Eq. (B27) out of the double sum $\sum_{n m}$ in Eq. (42). $g_{i / c}$ are kinematic functions of the order of unity. $\left|F_{p / n}\right|^{2}$ are proton and neutron form factors of the nucleus defined by Eq. (B14).

The first and second lines of Eq. (44) correspond to inelastic and elastic neutrino-nucleus scattering, respectively. Their dependencies on the number of nucleons are linear and quadratic, respectively. Using the terminology of Freedman, one would refer to these terms as incoherent and coherent, correspondingly.

This is the most general result of this work if terms with covariances defined in Eqs. (B31) and (B35) are neglected.

The summation of amplitudes due to the scattering off of various targets is evident in the second line of Eq. (44). Each type of nucleon is weighted according to the appropriate averaged form factor $F_{p / n}(\boldsymbol{q})$. Note, that the nucleus does not change its spin eigenstate in the coherent term. This is encoded in the summation $\sum_{r}\left(l, h_{r r}^{p / n}\right)$.

The incoherent term depends on $\left|\lambda_{s r}^{p / n}\right|^{2}$. The latter is a probability for a nucleon to change spin index $r$ to $s$ in transitions $|n\rangle \rightarrow|m\rangle$, averaged over $n$ and summed up over $m$.

While one needs a model for the nucleus wave functions to calculate $\left|\lambda_{s r}^{p / n}\right|^{2}$, we approximate these coefficients by unity $\left|\lambda_{s r}^{p / n}\right|^{2} \rightarrow 1$, which implies that for any $r$, any value of 
$s$ is possible with the same probability. Therefore, we can complete our calculations of the cross section.

The scalar products $\left(l, h^{p / n}\right)$ are calculated in Appendix C using helicity and $\sigma_{3}$ bases. The latter corresponds to the basis with spin projection on a fixed axis chosen to be along the incoming neutrino momentum. While the results do not depend on the basis chosen, as demonstrated in Eq. (C39), it is more straightforward to use the helicity basis with Eq. (C12) and $\sigma_{3}$ basis with Eq. (C33) to calculate the incoherent and coherent cross sections, respectively.

As follows from Eq. (44), the observable neutrinonucleus cross section can be presented as a sum of incoherent and coherent cross sections

$$
\frac{d \sigma}{d T_{A}}=\frac{d \sigma_{\mathrm{incoh}}}{d T_{A}}+\frac{d \sigma_{\mathrm{coh}}}{d T_{A}}
$$

The incoherent cross section reads

$$
\begin{aligned}
\frac{d \sigma_{\text {incoh }}}{d T_{A}}= & \frac{2 G_{F}^{2} m_{A}}{\pi} g_{i} \sum_{f=n, p}\left(1-\left|F_{f}\right|^{2}\right) \\
& \times\left[A_{f}\left(g_{L, f}^{2}+g_{R, f}^{2}(1-y)^{2}-2 g_{L, f} g_{R, f} \frac{y m_{N}^{2}}{s-m_{N}^{2}}\right)\right. \\
& +\Delta A_{f}\left(\left[g_{L, f}-g_{R, f}(1-y)\right]\right. \\
& \left.\left.\cdot\left[g_{L, f}+g_{R, f}\left(1-y \frac{s+m_{N}^{2}}{s-m_{N}^{2}}\right)\right]\right)\right],
\end{aligned}
$$

where $g_{L}^{p / n}$ and $g_{R}^{p / n}$ are left- and right-chirality couplings of the nucleons expressed via corresponding vector and axial couplings in Eq. (B9). The Bjorken $y$ is defined in Eq. (C13). The total energy squared $s=(p+k)^{2}$ of the neutrino and target nucleon is calculated assuming an effective momentum of the nucleon given by Eq. (33). Let us note that $y$ and $s$ are determined within neutrinonucleon kinematics.

In Eq. (46), $A_{p}=Z, A_{n}=N$ and $\Delta A_{p} \equiv \Delta Z=Z_{+}-Z_{-}$, $\Delta A_{n} \equiv \Delta N=N_{+}-N_{-}$, where $Z_{ \pm}, N_{ \pm}$stand for the numbers of protons and neutrons with spin projection on the incident neutrino momentum axis equal to $\pm 1 / 2$. A correction function $g_{i}^{p / n}$ of the order of unity is discussed earlier and is defined in Eq. (B27).

If the target nuclei are unpolarized, then terms proportional to $\Delta A^{f}$ in Eq. (46) vanish after averaging. Therefore, for an unpolarized target the incoherent cross section reads

$$
\begin{aligned}
\frac{d \sigma_{\text {incoh }}}{d T_{A}}= & \frac{2 G_{F}^{2} m_{A}}{\pi} g_{i} \sum_{f=n, p}\left(1-\left|F_{f}\right|^{2}\right) \\
& \times A^{f}\left(g_{L, f}^{2}+g_{R, f}^{2}(1-y)^{2}-2 g_{L, f} g_{R, f} \frac{y m_{N}^{2}}{s-m_{N}^{2}}\right) .
\end{aligned}
$$

The coherent cross section reads

$$
\frac{d \sigma_{\mathrm{coh}}}{d T_{A}}=\frac{G_{F}^{2} m_{A}}{\pi} g_{c}\left(1-\frac{T_{A}}{T_{A}^{\max }}\right)\left|G_{V}+G_{A}\right|^{2},
$$

where

$$
\begin{aligned}
G_{V} & =\sum_{f} g_{V}^{f} F_{f}\left(A_{f}\left[1-\frac{y \tau}{2}\right]+\Delta A_{f} \frac{y}{2}\right), \\
G_{A} & =\sum_{f} g_{A}^{f} F_{f}\left(\Delta A_{f}\left[1-\frac{y}{2}\right]+A_{f} \frac{y \tau}{2}\right),
\end{aligned}
$$

where

$$
\tau=\frac{\sqrt{s}-m_{N}}{\sqrt{s}+m_{N}} .
$$

It is straightforward to perform the spin averaging in Eq. (48), removing the terms linear in $\Delta A_{f}$. The final formula of the spin-averaged cross section reads

$$
\begin{aligned}
\frac{d \sigma_{\mathrm{coh}}}{d T_{A}}= & \frac{G_{F}^{2} m_{A}}{\pi} g_{c}\left(1-\frac{T_{A}}{T_{A}^{\max }}\right) \sum_{f, f^{\prime}} F_{f} F_{f^{\prime}}^{*} \\
& \times\left[g_{V}^{f} g_{V}^{f^{\prime}}\left(A_{f} A_{f^{\prime}}\left(1-\frac{y \tau}{2}\right)^{2}+\Delta A_{f} \Delta A_{f^{\prime}}\left(\frac{y}{2}\right)^{2}\right)\right. \\
& +g_{A}^{f} g_{A}^{f^{\prime}}\left(\Delta A_{f} \Delta A_{f^{\prime}}\left(1-\frac{y}{2}\right)^{2}+A_{f} A_{f^{\prime}}\left(\frac{y \tau}{2}\right)^{2}\right) \\
& +2 g_{V}^{f} g_{A}^{f^{\prime}}\left(A_{f} A_{f^{\prime}}\left(1-\frac{y \tau}{2}\right) \frac{y \tau}{2}+\Delta A_{f} \Delta A_{f^{\prime}} \frac{y}{2}\left(1-\frac{y}{2}\right)\right] .
\end{aligned}
$$

Finally, Eq. (51) could be simplified if the following approximations are adopted. (i) Terms proportional to $y \approx$ $3 \% E_{\nu} /(30 \mathrm{MeV})$ are omitted. (ii) Terms proportional to $\Delta A_{f} \Delta A_{f^{\prime}}$ are neglected. This can be done either for a spinless nucleus, or approximately for heavy nuclei with $\Delta A \ll A$. (iii) Terms proportional to $g_{V}^{p}$ are abandoned because $g_{V}^{p} \ll 1$. (iv) The kinematic correction function $g_{c} \rightarrow 1$.

Therefore,

$$
\frac{d \sigma_{\mathrm{coh}}}{d T_{A}} \approx \frac{G_{F}^{2} m_{A}}{\pi}\left(1-\frac{T_{A}}{T_{A}^{\max }}\right)\left|F_{n}\right|^{2}\left(g_{V}^{n}\right)^{2} N^{2},
$$

which is a well known result [1-5,7-10,20,32,42]. Corrections to this formula are discussed in Sec. III B.

\section{DISCUSSION}

In what follows, we discuss in detail the calculated cross section. It is convenient to refer to the cross section integrated over the kinetic energy of the recoil nucleus 


$$
\sigma\left(E_{\nu}\right)=\int_{T_{A}^{\min }}^{T_{A}^{\max }} \frac{d \sigma}{d T_{A}} d T_{A}
$$

This integral depends on the energy threshold $T_{A}^{\min }$, unique for each detector. As an illustration we consider three experimental setups.

We refer to the state-of-the-art energy thresholds of considered experimental setups, briefly described in what follows.

(i) A germanium detector exposed to $\bar{\nu}_{e}$ flux from a nuclear reactor. Among all natural isotopes we select only one stable nucleus, ${ }^{74} \mathrm{Ge}$, for our illustration. The expected energy threshold for electrons of germanium bolometers is $200 \mathrm{eV}$ [76], which, accounting for the quenching in germanium detectors [77], roughly corresponds to $1 \mathrm{keV}$ of the ${ }^{74} \mathrm{Ge}$ recoil kinetic energy. We refer to the $\nu$ GEN experiment at the Kalinin Nuclear Power Plant [50] as an example. For illustration, we calculate the differential cross sections for two $\bar{\nu}_{e}$ energies, 5 and $7 \mathrm{MeV}$, and total cross section for $E_{\nu} \in(1,20) \mathrm{MeV}$. As an estimate for an excitation energy of the ${ }^{74} \mathrm{Ge}$ nucleus we take $\Delta \varepsilon=900 \mathrm{keV}$.

(ii) A CsI scintillator exposed to the neutrinos from the Spallation Neutron Source [63]. The differential and total cross sections are calculated for $E_{\nu}=30$ and $50 \mathrm{MeV}$ and for $E_{\nu} \in(1,150) \mathrm{MeV}$, respectively. We assumed $\Delta \varepsilon=100 \mathrm{keV}$ for the ${ }^{133} \mathrm{Cs}$ nucleus. The energy threshold was set to $5 \mathrm{keV}$ of the ${ }^{133} \mathrm{Cs}$ recoil kinetic energy.

(iii) A liquid argon detector with an unprecedented low-energy threshold of $0.6 \mathrm{keV}$ for the ${ }^{40} \mathrm{Ar}$ nucleus achieved by the DarkSide Collaboration [78]. The differential and total cross sections are calculated for $E_{\nu}=15 \mathrm{MeV}$ and for $E_{\nu} \in(1,50) \mathrm{MeV}$, respectively.

To make a prediction for an experiment we use (i) two form factors $F_{p / n}(\boldsymbol{q})$ for protons and neutrons, respectively, and (ii) data regarding the energy levels of the nucleus under consideration.

We considered two models of the form factors: symmetrized Fermi-distribution [79] and Helm form factor [80]. Both models of the form factors give very similar results numerically if the parameters of the models are selected to reproduce the same proton and neutron RMS radii. In what follows, we present the results obtained assuming the same RMS radii for protons and neutrons, and using the Helm form factors for definiteness.

In Fig. 5, predictions of these models as functions of $|\boldsymbol{q}|$ are depicted. At $T_{A} \simeq(12-15) \mathrm{keV}$, where the maximum of the signal observed by the COHERENT experiment occurred, $|\boldsymbol{q}| \simeq(50-60) \mathrm{MeV}$ and $|F(\boldsymbol{q})|^{2} \simeq(0.6-0.5)$, indicating that pure coherent scattering has a suppression and a contribution from the incoherent transitions should be expected.

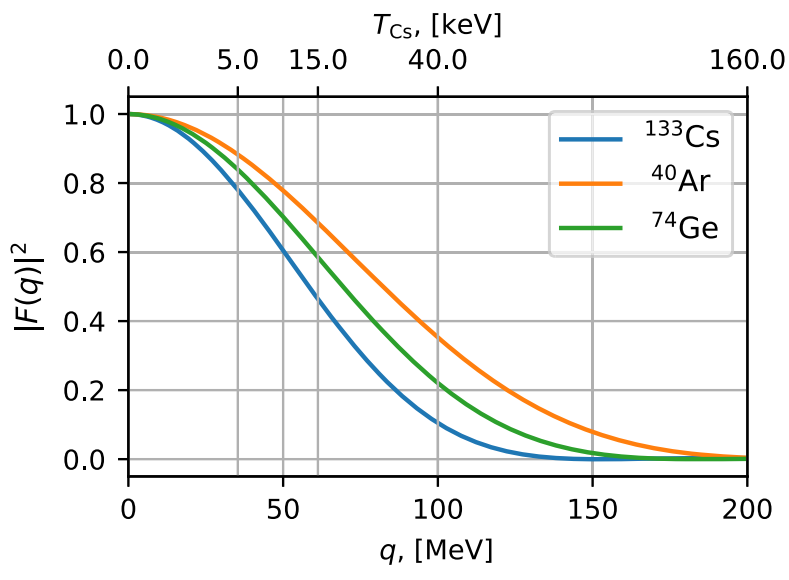

FIG. 5. The Helm form factor $F^{\text {Helm }}$ [80] as a function of the absolute value of three-momentum transfer $|\boldsymbol{q}|$ (bottom horizontal axis). The upper horizontal axis corresponds to the kinetic energy of ${ }^{133}$ Cs nucleus.

\section{A. Coherent and incoherent}

The most general feature of Eq. (45) consists of smooth transitions between coherent and incoherent regimes. Both terms of the cross section are governed by the same $F_{p / n}(\boldsymbol{q})$ form factors defined in Eq. (B28).

In the limit $\boldsymbol{q} \rightarrow 0, F_{p / n}(\boldsymbol{q}) \rightarrow 1$, and the contribution of the incoherent cross section vanishes, while the coherent term totally dominates.

In the opposite limit of large $\boldsymbol{q}$, when $F_{p / n}(\boldsymbol{q}) \rightarrow 0$, the coherent cross section vanishes and the incoherent term dominates. In general, both coherent and incoherent scatterings contribute.

In Fig. 6, the differential coherent and incoherent cross sections are displayed for three experimental setups discussed above.

(i) At $T_{A} \rightarrow 0$, the coherent cross section totally dominates since the incoherent contribution vanishes. For a given nucleus, the coherent differential cross section in this limit does not depend on neutrino energy up to small corrections, in agreement with Eq. (48).

(ii) At $T_{A} \rightarrow T_{A}^{\max }$, the coherent cross section vanishes because of the factor $1-T_{A} / T_{A}^{\max }$, while the incoherent cross section rises. One might observe that the maximum kinetic energy of the nucleus experienced in an incoherent scattering is systematically smaller than that for the coherent interaction. This is because some of the neutrino energy is used for the excitation of the nucleus, as given by Eq. (29).

(iii) For small neutrino energies, the coherent cross section dominates over the incoherent contribution for any $T_{A}$. For larger $E_{\nu}$, there is a value of $T_{A}$ above which the incoherent cross section dominates over the coherent, as can be seen in the middle panel of Fig. 6 for $E_{\nu}=50 \mathrm{MeV}$. In particular, for 

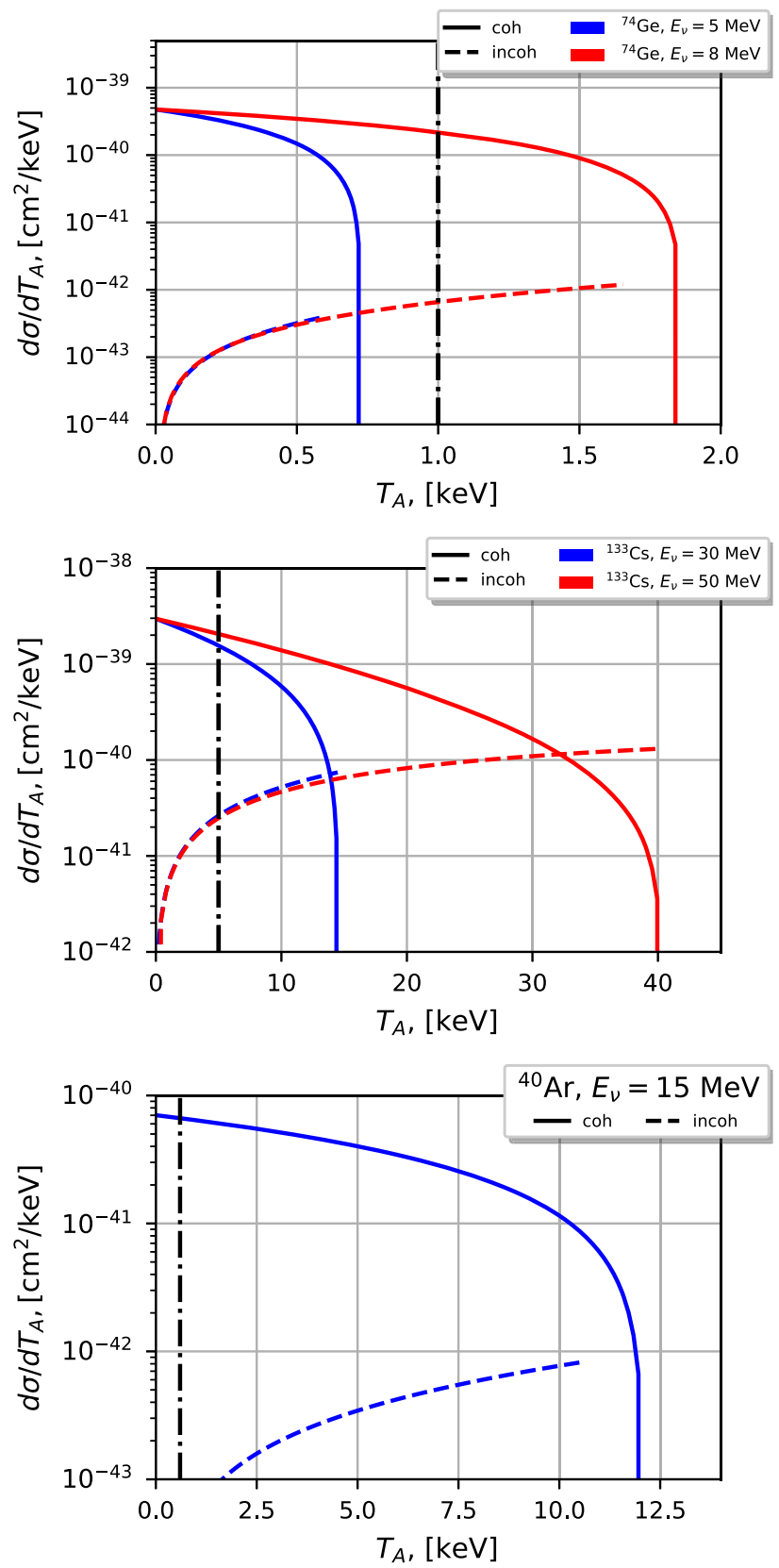

FIG. 6. Differential cross sections $\frac{d \sigma}{d T_{A}}$ for coherent (solid lines) and incoherent (dashed lines) neutrino-nucleus scattering for ${ }^{74} \mathrm{Ge}$ (top), ${ }^{133} \mathrm{Cs}$ (middle) and ${ }^{40} \mathrm{Ar}$ (bottom) nuclei and different values of neutrino energies. Vertical lines correspond to experimental energy thresholds.

$E_{\nu}=50 \mathrm{MeV}$ with a ${ }^{133} \mathrm{Cs}$ nucleus this occurs at $T_{A} \gtrsim 33 \mathrm{keV}$.

In Fig. 7, the corresponding integral cross sections are displayed.

(i) At low $E_{\nu}$, the coherent integral cross section is larger than the incoherent by orders of magnitude because the factors $1-\left|F_{p / n}(\boldsymbol{q})\right|^{2}$ suppress the latter at small $\boldsymbol{q}$. With increasing neutrino energy their interrelation changes to the exact opposite, the
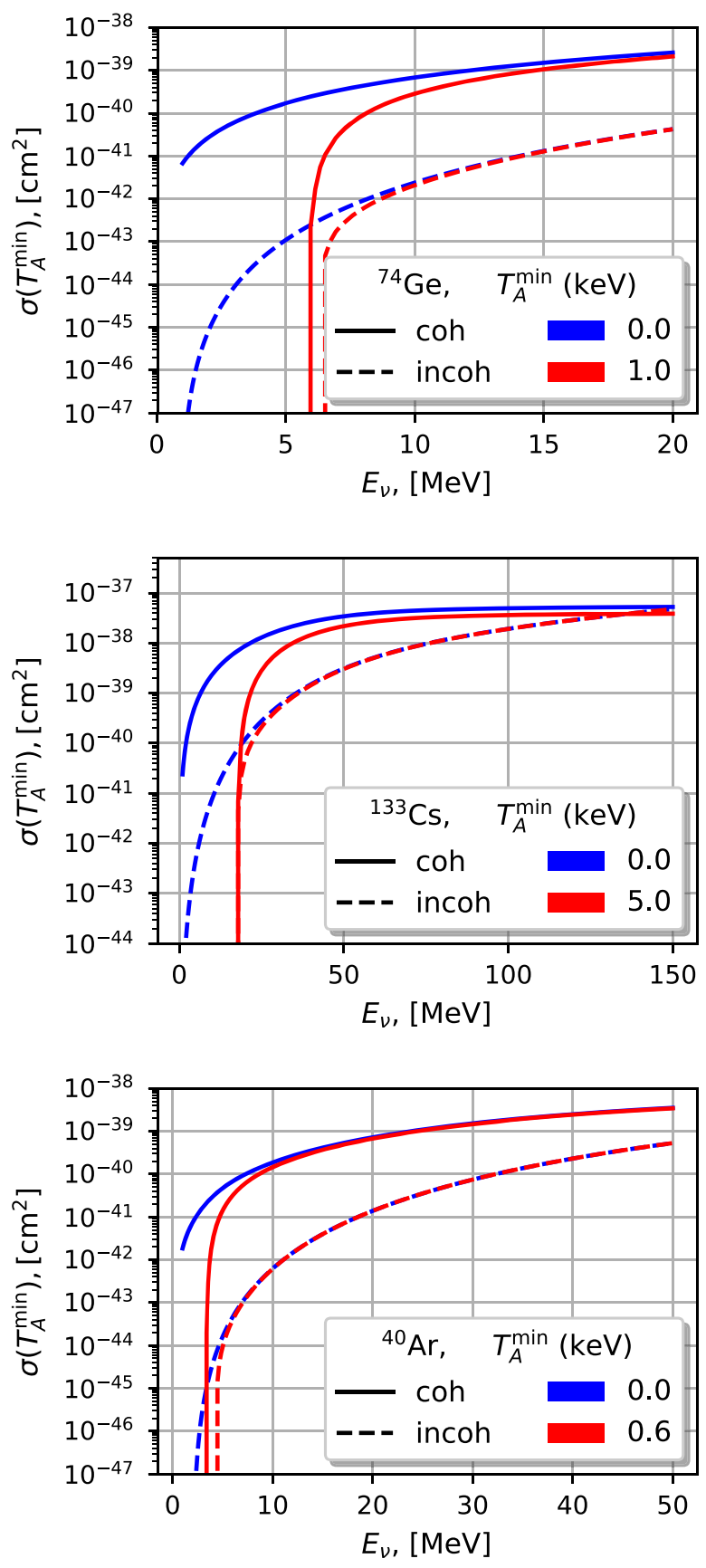

FIG. 7. Integral cross sections $\sigma$ for coherent (solid lines) and incoherent (dashed lines) neutrino-nucleus scattering for ${ }^{74} \mathrm{Ge}$ (top), ${ }^{133} \mathrm{Cs}$ (middle), and ${ }^{40} \mathrm{Ar}$ (bottom) nuclei and different values of neutrino energies. The integrals are calculated for idealistic threshold-less $\left(T_{A}^{\min }=0\right.$, blue lines) experimental setups and accounting for state-of-the-art thresholds $T_{A}^{\min }$ (red lines) achieved by three considered experimental setups.

incoherent cross section dominating above a certain $E_{\nu}$. As an example, for the ${ }^{133} \mathrm{Cs}$ nucleus this occurs at $E_{\nu} \gtrsim 140(120) \mathrm{MeV}$ for $T_{A}^{\min }=0(5) \mathrm{keV}$.

(ii) The experimental detection threshold reduces the integrated coherent cross section and, to a lesser extent the incoherent, because the threshold removes 


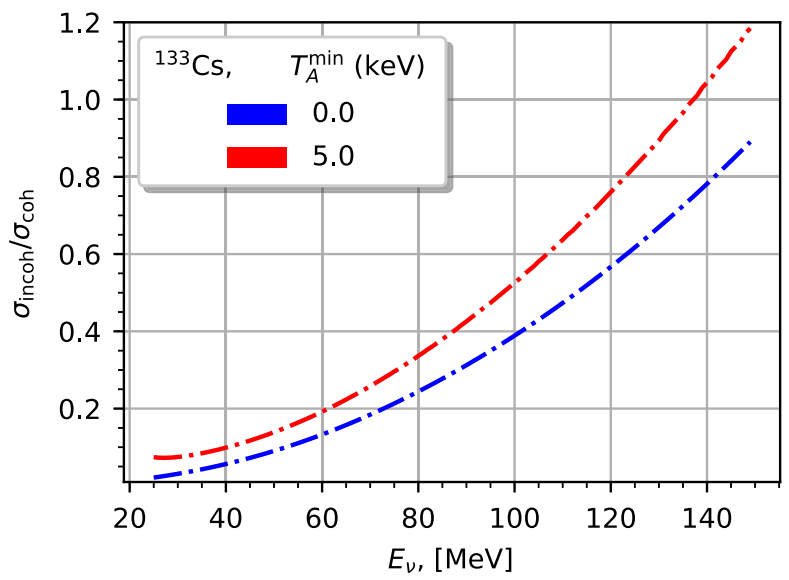

FIG. 8. Ratio $\sigma_{\text {incoh }} / \sigma_{\text {coh }}$ for neutrino scattering off of a ${ }^{133} \mathrm{Cs}$ nucleus as a function of $E_{\nu}$. The two curves correspond to a $T_{A}^{\min }=0(5) \mathrm{keV}$ detection threshold.

the part of the differential cross section which is the largest for the former and vanishing for the latter, as can be seen in Fig. 6. To quantify this statement the ratio of integrals given by Eq. (53), $\sigma_{\text {incoh }} / \sigma_{\text {coh }}$, is displayed in Fig. 8 for the ${ }^{133} \mathrm{Cs}$ nucleus. At $E_{\nu} \simeq 30(50) \mathrm{MeV}$, this ratio is about $5(10) \%$ for an idealistic thresholdless experiment, and reaches about $10(20) \%$ for $T_{A}^{\min }=5 \mathrm{keV}$. The increasing importance of the incoherent interaction is evident for increasing neutrino energy.

\section{B. Revising the coherent cross section}

It is instructive to compare the coherent cross section in Eq. (48) and (51) to that used in the literature [65]

$$
\begin{aligned}
\frac{d \sigma_{\mathrm{coh}}^{0}}{d T_{A}}= & \frac{G_{F}^{2} m_{A}}{2 \pi}\left[\left(G_{V}+G_{A}\right)^{2}+\left(G_{V}-G_{A}\right)^{2}(1-y)^{2}\right. \\
& \left.-\left(G_{V}^{2}-G_{A}^{2}\right) \frac{m_{A} T_{A}}{E_{\nu}^{2}}\right] \\
\approx & \frac{G_{F}^{2} m_{A}}{\pi}\left[G_{V}^{2}\left(1-\frac{T_{A}}{T_{A}^{\max }}\right)+G_{A}^{2}\left(1+\frac{T_{A}}{T_{A}^{\max }}\right)\right] \\
\approx & \frac{G_{F}^{2} m_{A}}{\pi}\left(1-\frac{T_{A}}{T_{A}^{\max }}\right)\left|F_{n}\right|^{2}\left(g_{V}^{n}\right)^{2} N^{2}
\end{aligned}
$$

where $G_{V}=F_{p} Z g_{V}^{p}+F_{n} N g_{V}^{n}$ and $G_{A}=F_{p} \Delta Z g_{A}^{p}+F_{n} \Delta N g_{A}^{n}$.

The second approximate equality of Eq. (54) appeared as a result of a quite accurate approximation $y=T_{A} / E_{\nu} \rightarrow 0$. The last line is a result of further approximations: (i) $g_{V}^{p} \rightarrow 0$ and (ii) spin-less nucleus.

Let us briefly review Eq. (54). After a number of approximations, the third line of Eq. (54) is identical to an approximation of the coherent cross section in Eq. (52), calculated in this work. However, conceptually, a derivation of Eq. (54) is at odds with the coherency. Indeed, as one can

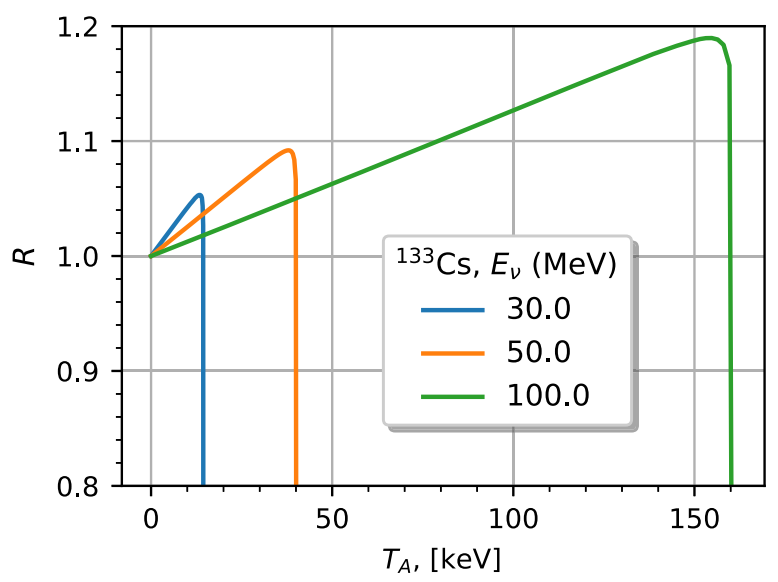

FIG. 9. Ratio $R$ of differential coherent cross section $d \sigma / d T_{A}$ calculated in this work in Eq. (48) to that used by the COHERENT Collaboration and reproduced in Eq. (54). Both cross sections are averaged over the nucleus spin, assuming neutrino scattering off of a ${ }^{133} \mathrm{Cs}$ nucleus. The ratio is shown as a function of kinetic energy $T_{A}$ of the nucleus.

observe, the first line of Eq. (54) corresponds to a calculation of incoherent cross section [compare to Eq. (47)], where the nucleus changes its spin eigenstate. As we advocate here, the coherent scattering corresponds to interactions of neutrino with the nucleon in which the latter remains in the same quantum state.

How then are Eqs. (54) and (46) consistent with a good accuracy? The reason is in the nonrelativistic approximation. Two terms of the matrix element containing $\left(l, h_{+-}^{\eta}\right)$ and $\left(l, h_{-+}^{\eta}\right)$ with a spin-flip should not contribute to the coherent cross section [on the opposite, they do exist in Eq. (54)]. In the nonrelativistic approximation, $\left(l, h_{+-}^{\eta}\right)$ vanishes, while $\left(l, h_{-+}^{\eta}\right)$ is proportional to $g_{A}$ and vanishes for a spin-less nucleus, as can be seen in Eq. (C35). The last statement is accurate if the nucleons in the nucleus are at rest.

To illustrate the effects of a moving target nucleon and constant spin of the nucleus in elastic neutrino-nucleus scattering, a ratio of differential coherent cross section $d \sigma / d T_{A}$ in Eq. (48) to that in Eq. (54) is displayed in Fig. 9 for a ${ }^{133} \mathrm{Cs}$ nucleus, assuming three fixed values of neutrino energy. The cross sections coincide at $T_{A}=0$ and show a difference at some percent with increasing $T_{A}$. The maximal difference occurring at the end of the nucleus kinetic energy spectra, rises with neutrino energy from about $5 \%$ at $E_{\nu}=30 \mathrm{MeV}$ to about $20 \%$ at $E_{\nu}=100 \mathrm{MeV}$.

There are two groups of corrections to the cross section of elastic neutrino-nucleus scattering.

(i) The most significant correction is due to $g_{c}$ normalization factor defined in Eq. (B27). The latter is a product of three functions, $C_{1}, C_{2}$ and $C_{3}$, determined by Eqs. (B19), (B23) and (B26). $C_{1}$ and $C_{2}$ functions are very close to unity, while $C_{3}$ rises linearly with the kinetic energy of the recoil nucleus. 
At $E_{\nu}=30,50$ and $100 \mathrm{MeV} C_{3}$ reaches about 6\%, $10 \%$, and $20 \%$ respectively.

(ii) Corrections to the vector and axial form factors defined in Eq. (49) are due to nonzero momentum of the target nucleon. The correction to $G_{V}$ is small, of the order $10^{-4}-10^{-3}$, because $\Delta A_{f}$ is multiplied by $y$-Bjorken which is small for elastic scattering. The correction to $G_{A}$ is of the order $10^{-2}-10^{-1}$ because a small factor $y \tau / 2$ is multiplied by a large factor $A_{f}$ making their product $A_{f} y \tau / 2$ at few percent of $\Delta A_{f}\left(1-\frac{y \tau}{2}\right)$.

\section{Proposal to observe higher energy excitation $\gamma s$ due to incoherent scattering}

After an interaction the nucleus may remain in the same quantum state, or the internal state of the nucleus could be changed. We refer to these cases as elastic and inelastic interactions. Experimentally, the scattered nucleus, being in the same or an excited state, are practically indistinguishable if one measures only the kinetic energy of the nucleus.

Inelastic interactions must be accompanied by the emission of gammas corresponding to the difference of energy levels of the nucleus. The time scale of these emissions is in the range of picoseconds to nanoseconds for the ${ }^{133} \mathrm{Cs}$ nucleus, taken as an example. The energies of the $\gamma \mathrm{s}$ are of the order of some hundred $\mathrm{keV}$ for the same nucleus. These $\gamma \mathrm{s}$ should produce a very detectable signal in the scintillator correlated in time with the beam pulses for an accelerator based experiment. The rate of these $\gamma \mathrm{s}$ is determined by the ratio $N_{\text {inc }} / N_{\text {coh }}$, where

$N_{\text {incoh } / \text { coh }}=\int d E_{\nu} \Phi\left(E_{\nu}\right) \int_{d T_{A}^{\min }}^{d T_{A}^{\max }} d T_{A} \frac{d \sigma_{\mathrm{inc} / \mathrm{coh}}}{d T_{A}} \varepsilon\left(T_{A}\right)$,

in which $\varepsilon\left(T_{A}\right)$ is the detection efficiency. Fig. 8 suggests that the number of $\gamma$ events due to incoherent interactions should be detectable.

It is remarkable, that a similar proposal was made back to 1975 in [74].

\section{SUMMARY}

A theoretical framework for neutrino-nucleus scattering $\nu A \rightarrow \nu A$, in which the nucleus conserves its integrity, is developed. The main result of this work consists in the demonstration that coherent and incoherent regimes appear due to elastic and inelastic processes, when all possible initial and final states are taken into account. This conclusion is in agreement with corresponding theories of scattering of $\mathrm{x}$ rays and electrons of an atom, and of slow neutrons off matter constituents.
The coherent and incoherent cross sections were shown to be driven by $\left|F_{p / n}\right|^{2}$ and $\left(1-\left|F_{p / n}\right|^{2}\right)$ factors, thus providing a smooth transition between these regimes.

We also revised a formula for the coherent cross section. The obtained formula has some percent level corrections when compared to that known in the literature (see, e.g., in [65]). They differ at most at the end of kinetic energy spectrum of the target nucleus, reaching $\approx 5 \%$ at $E_{\nu}=$ $30 \mathrm{MeV}\left(\approx 20 \%\right.$ at $\left.E_{\nu}=100 \mathrm{MeV}\right)$. There are two main sources for this difference. (i) Our consideration treats only those matrix elements which correspond to the same initial and final spin states of the nucleus in contrast to the conventional derivation which considers also the spinflipped matrix elements. (ii) The target nucleon is not assumed to be at rest which develops corrections to the vector and axial form factors of the nucleus.

Three experimental setups considered in this work illustrate our results. In particular, for ${ }^{133} \mathrm{Cs}$ and neutrino energies of $30-50 \mathrm{MeV}$ the incoherent cross section is about $10 \%-20 \%$ of the coherent contribution if experimental detection threshold is accounted for. The incoherent processes being a relatively small "background" to the coherent interactions provide an important clue if $\gamma \mathrm{s}$ released by excited nucleus are detected. Detection of both signals due to nuclear recoil and excitations $\gamma \mathrm{s}$ provides a more sensitive instrument in studies of nuclear structure and possible signs of new physics.

An interested reader could checkout and run a Jupyter Notebook where equations from this manuscript are documented in terms of a python code [81].

\section{ACKNOWLEDGMENTS}

We are grateful to D. Dwyer, C. Giunti, M. Gonchar, S. E. Korenblit, C. Kullenberg, V. A. Kuzmin, J. Link, V. A. Naumov, O. Yu. Smirnov, E. Yakushev, S. Zhou, and X. Qian for reading the manuscript and making important comments. The authors are thankful to Joint Institute for Nuclear Research for constant support during completion of this work.

\section{APPENDIX A: DECOMPOSITION OF $n$-PARTICLE STATES IN $x$ AND $p$ BASES}

In this section, we shortly summarize some mathematical aspects of the representations of abstract quantum states for both single fermion and $n$-fermions.

\section{Single-particle states}

We begin by reminding the reader about the singleparticle basis. A fermionic state with mass $m$, definite threemomentum $\boldsymbol{p}$, energy $E_{\boldsymbol{p}}=\sqrt{\boldsymbol{p}^{2}+m^{2}}$ and spin projection $s$ is defined according to

$$
|\boldsymbol{p}, s\rangle=\sqrt{2 E_{p}} a_{p, s}^{\dagger}|0\rangle,
$$


with Lorentz-invariant normalization

$$
\langle\boldsymbol{k}, s \mid \boldsymbol{p}, r\rangle=(2 \pi)^{3} 2 E_{\boldsymbol{p}} \delta^{3}(\boldsymbol{p}-\boldsymbol{k}) \delta_{r s} .
$$

A fermionic state with definite $\boldsymbol{x}$ can be defined as

$$
\langle\boldsymbol{x}|=\langle 0| \hat{\psi}(\boldsymbol{x}),
$$

where $\hat{\psi}(\boldsymbol{x})$ is the free field operator in the Schroedinger representation. The state in Eq. (A3) is a Dirac spinor. These states are normalized as follows

$$
\langle\boldsymbol{y} \mid \boldsymbol{x}\rangle=\delta^{3}(\boldsymbol{x}-\boldsymbol{y}) \hat{I}_{4 \times 4},
$$

where $\hat{I}_{4 \times 4}$ is the $4 \times 4$ unity matrix in the spinor space.

The single-particle unity operators read

$$
\begin{aligned}
& \hat{I}_{p, 1}=\int \frac{d \boldsymbol{p}}{(2 \pi)^{3}} \frac{|\boldsymbol{p}, s\rangle\langle\boldsymbol{p}, s|}{2 E_{\boldsymbol{p}}}, \\
& \hat{I}_{x, 1}=\int d \boldsymbol{x}|\boldsymbol{x}\rangle\langle\boldsymbol{x}|
\end{aligned}
$$

The scalar product of states given by Eqs. (A1) and (A3)

$$
\langle\boldsymbol{x} \mid \boldsymbol{p}, s\rangle=u(\boldsymbol{p}, s) e^{i \boldsymbol{p} x}
$$

allows for the representation of $|\boldsymbol{p}, s\rangle$ and $|\boldsymbol{x}\rangle$ states via linear superpositions of each other

$$
\begin{aligned}
\langle\boldsymbol{p}, s| & =\int d \boldsymbol{x} u^{\dagger}(\boldsymbol{p}, s) e^{-i p x}\langle\boldsymbol{x}|, \\
\langle\boldsymbol{x}| & =\int \frac{d \boldsymbol{p}}{(2 \pi)^{3}} \frac{u(\boldsymbol{p}, s) e^{i p x}}{2 E_{\boldsymbol{p}}}\langle\boldsymbol{p}, s| .
\end{aligned}
$$

The second line of Eq. (A7) allows us to see that $\langle\boldsymbol{x}|$, given by Eq. (A3), differs from a nonrelativistic spin independent state

$$
\langle\mathbf{x}|=\int \frac{d \boldsymbol{p}}{(2 \pi)^{3}} \frac{e^{i x p}}{\sqrt{2 E_{p}}}\langle\boldsymbol{p}|,
$$

where $\langle\boldsymbol{p}|$ is defined similarly to Eq. (A1) but for a spin-less particle.

A one-particle state $|\psi\rangle$ can be represented through $|\boldsymbol{p}, s\rangle$ and $|\boldsymbol{x}\rangle$ states

$$
\begin{aligned}
|\psi\rangle \equiv \hat{I}_{p, 1}|\psi\rangle & =\int \frac{d \boldsymbol{p}}{(2 \pi)^{3}}|\boldsymbol{p}, s\rangle \frac{\tilde{\psi}(\boldsymbol{p}, s)}{\sqrt{2 E_{\boldsymbol{p}}}}, \\
|\psi\rangle \equiv \hat{I}_{x, 1}|\psi\rangle & =\int d \boldsymbol{x}|\boldsymbol{x}\rangle \psi(\boldsymbol{x}),
\end{aligned}
$$

where $\tilde{\psi}(\boldsymbol{p}, s)=\langle\boldsymbol{p}, s \mid \psi\rangle / \sqrt{2 E_{\boldsymbol{p}}}$ and $\psi(\boldsymbol{x})=\langle\boldsymbol{x} \mid \psi\rangle$.
Imposing $\langle\psi \mid \psi\rangle=1$ the wave functions $\tilde{\psi}(\boldsymbol{p}, s)$ and $\psi(\boldsymbol{x})$ are normalized according to

$$
\int \frac{d \boldsymbol{p}}{(2 \pi)^{3}}|\tilde{\psi}(\boldsymbol{p}, s)|^{2}=\int d \boldsymbol{x}|\psi(\boldsymbol{x})|^{2}=1 .
$$

These wave functions are related to each other through the Fourier transform

$$
\begin{aligned}
\psi(\boldsymbol{x}) & =\int \frac{d \boldsymbol{p}}{(2 \pi)^{3}} \frac{\tilde{\psi}(\boldsymbol{p}, s)}{\sqrt{2 E_{\boldsymbol{p}}}} u(\boldsymbol{p}, s) e^{i \boldsymbol{p} \boldsymbol{x}}, \\
\tilde{\psi}(\boldsymbol{p}, s) & =\frac{u^{\dagger}(\boldsymbol{p}, s)}{\sqrt{2 E_{\boldsymbol{p}}}} \int d \boldsymbol{x} \psi(\boldsymbol{x}) e^{-i p x} .
\end{aligned}
$$

Note that $\tilde{\psi}(\boldsymbol{p}, s)$ is a scalar, while $\psi(\boldsymbol{x})$ is a Dirac spinor.

\section{2. $n$-particle states}

The unity operators defined in Eq. (A5), generalized for $n$-particle states, reads

$$
\begin{aligned}
& \hat{I}_{p, n}=\int\left(\prod_{i=1}^{n} \frac{d \boldsymbol{p}_{i}}{(2 \pi)^{3} 2 E_{\boldsymbol{p}_{i}}}\right) \frac{|\{p\}\rangle\langle\{p\}|}{n !} \\
& \hat{I}_{x, n}=\int\left(\prod_{i=1}^{n} d \boldsymbol{x}_{i}\right) \frac{|\{x\}\rangle\langle\{x\}|}{n !} .
\end{aligned}
$$

The symbols $\{p\}$ and $\{x\}$ are $n$-tuples, $\{p\}=\left(p_{1} \ldots p_{n}\right)$ and $\{x\}=\left(x_{1} \ldots x_{n}\right)$ are used for compaction here and in what follows.

The bra-vector $\langle\{x\}|$ is given as

$$
\langle\{x\}|=\langle 0| \psi_{m_{1}}\left(\boldsymbol{x}_{1}\right) \ldots \psi_{m_{n}}\left(\boldsymbol{x}_{n}\right),
$$

with $x_{i}=\left(\boldsymbol{x}_{i}, m_{i}\right)$, where $m_{i}$ enumerates the spinor's rows of the fields $\psi\left(\boldsymbol{x}_{i}\right)$.

Similarly to Eq. (A9) the wave functions in both momentum and coordinate spaces for the $n$-particle state $|\psi\rangle$ can be obtained using $|\psi\rangle=\hat{I}_{p, n}|\psi\rangle=\hat{I}_{x, n}|\psi\rangle$

$$
\begin{aligned}
& |\psi\rangle=\int\left(\prod_{i=1}^{n} d \tilde{\boldsymbol{p}}_{i}\right) \frac{\tilde{\psi}(\{p\})}{\sqrt{n !}}|\{p\}\rangle, \\
& |\psi\rangle=\int\left(\prod_{i=1}^{n} d \boldsymbol{x}_{i}\right) \frac{\psi(\{x\})}{\sqrt{n !}}|\{x\}\rangle,
\end{aligned}
$$

where

$$
\begin{aligned}
& \tilde{\psi}(\{p\})=\frac{\langle\{p\} \mid \psi\rangle}{\sqrt{n !} \prod_{i} \sqrt{2 E_{p_{i}}}}, \\
& \psi(\{x\})=\frac{1}{\sqrt{n !}}\langle\{x\} \mid \psi\rangle
\end{aligned}
$$

and 


$$
d \tilde{\boldsymbol{p}}_{i} \equiv \frac{d \boldsymbol{p}_{i}}{(2 \pi)^{3} \sqrt{2 E_{\boldsymbol{p}_{i}}}}
$$

The wave functions $\tilde{\psi}(\{p\})$ and $\psi(\{x\})$ are Fourier transforms of each other

$$
\begin{gathered}
\psi(\{x\})=\int\left(\prod_{i=1}^{n} d \tilde{\boldsymbol{p}}_{i} u_{m_{i}}\left(\boldsymbol{p}_{i}, s_{i}\right) e^{i \boldsymbol{p}_{i} \boldsymbol{x}_{i}}\right) \tilde{\psi}(\{p\}), \\
\tilde{\psi}(\{p\})=\int\left(\prod_{i=1}^{n} d \boldsymbol{x}_{i} \frac{u_{m_{i}}^{\dagger}\left(\boldsymbol{p}_{i}, s_{i}\right)}{\sqrt{2 E_{\boldsymbol{p}_{i}}}} e^{-i \boldsymbol{p}_{i} \boldsymbol{x}_{i}}\right) \psi(\{x\}) .
\end{gathered}
$$

Imposing $\langle\psi \mid \psi\rangle=1$ the wave functions $\tilde{\psi}(\{p\})$ and $\psi(\{x\})$ are normalized as

$\int\left(\prod_{i=1}^{n} \frac{d \boldsymbol{p}_{i}}{(2 \pi)^{3}}\right)|\tilde{\psi}(\{p\})|^{2}=\int\left(\prod_{i=1}^{n} d \boldsymbol{x}_{i}\right)|\psi(\{x\})|^{2}=1$.

Both $\tilde{\psi}(\{p\})$ and $\psi(\{x\})$ are anti-symmetric under an odd number of particle interchanges.

\section{The wave function of a nucleus}

The Fock state $\left|P_{n}\right\rangle$ of a nucleus, with four-momentum $P_{n}$ being in the $n$-th quantum state, can be written as a superposition of free nucleon states using their bound state wave function in the momentum representation $\tilde{\psi}_{n}^{\prime}$

$$
\left|P_{n}\right\rangle=\int\left(\prod_{i=1}^{A} d \tilde{\boldsymbol{p}}_{i}\right) \frac{\tilde{\psi}_{n}^{\prime}(\{p\})}{\sqrt{A !}}|\{p\}\rangle .
$$

The wave function $\tilde{\psi}_{n}^{\prime}(\{p\})$ describes both the internal structure of the nucleus and its movement as a whole with three-momentum $\boldsymbol{p}=\sum_{i=1}^{A} \boldsymbol{p}_{i}$ and spin projection $s$. Since the quantum state of $A$ interacting nucleons cannot depend on the motion of their center-of-mass, the wave function $\tilde{\psi}_{n}^{\prime}(\{p\})$ can be factorized into a product of the wave function $\tilde{\psi}_{n}\left(\left\{p^{\star}\right\}\right)$, describing the internal structure of the nucleus in its center-of-mass (the corresponding momenta are labeled by the upper index $\star$ ), and the wave function $\Phi(p)$, describing the motion of the nucleus with momentum $\boldsymbol{p}$ and spin projection $s$, both encoded in the argument $p$ of $\Phi$

$$
\tilde{\psi}_{n}^{\prime}(\{p\})=\tilde{\psi}_{n}\left(\left\{p^{\star}\right\}\right) \Phi_{n}(p) .
$$

The factorization in Eq. (A19) makes sense for $A>1$.

The three-momentum of the $i$ th nucleon in the center-ofmass frame is given by $\boldsymbol{p}_{i}^{\star}$. The $i$ th nucleon's momentum $\boldsymbol{p}_{i}$ in the laboratory system is given by

$$
\boldsymbol{p}_{i}=\boldsymbol{p}_{i}^{\star}+\boldsymbol{p} / A .
$$

The state in Eq. (A18) can now be rewritten as

$$
\left|P_{n}\right\rangle=\int\left(\prod_{i=1}^{A} d \tilde{\boldsymbol{p}}_{i}^{\star}\right) \frac{\psi_{n}\left(\left\{p^{\star}\right\}\right)}{\sqrt{A !}} \Phi_{n}(p)|\{p\}\rangle .
$$

We take the wave function $\Phi(p)$ of the form

$$
\Phi_{n}(p)=(2 \pi)^{3} \sqrt{2 P_{n}^{0}} \delta^{3}(\boldsymbol{p}-\boldsymbol{P}),
$$

which corresponds to a nucleus with a definite momentum $\boldsymbol{P}$ and energy $P_{n}^{0}=E_{\boldsymbol{p}}+\varepsilon_{n}$, including excitation energy $\varepsilon_{n}$. Then, the state in Eq. (A21) is normalized similarly to Eq. (A2)

$$
\left\langle P_{m}^{\prime} \mid P_{n}\right\rangle=(2 \pi)^{3} 2 P_{n}^{0} \delta^{3}\left(\boldsymbol{P}-\boldsymbol{P}^{\prime}\right) \delta_{n m}
$$

if the following normalization of the internal nucleus state $|n\rangle$ is adopted

$$
\begin{aligned}
\langle m \mid n\rangle= & \delta_{n m} \\
= & \int\left(\prod_{i=1}^{A} \frac{d \boldsymbol{p}_{i}^{\star}}{(2 \pi)^{3}}\right) \tilde{\psi}_{n}\left(\left\{p^{\star}\right\}\right) \tilde{\psi}_{m}^{*}\left(\left\{p^{\star}\right\}\right) \\
& \times(2 \pi)^{3} \delta^{3}\left(\sum_{i=1}^{A} \boldsymbol{p}_{i}^{\star}\right) .
\end{aligned}
$$

The delta-function $\delta^{3}\left(\sum_{i=1}^{A} \boldsymbol{p}_{i}^{\star}\right)$ reduces the number of independent momenta in Eq. (A23) by one.

The states $|n\rangle$ and $\left|P_{n}\right\rangle$ describe the same realm at $\boldsymbol{P}=0$ yet still differ by normalization. We define the former as

$|n\rangle=\int\left(\prod_{i=1}^{A} d \tilde{\boldsymbol{p}}_{i}^{\star}\right) \frac{\psi_{n}\left(\left\{p^{\star}\right\}\right)}{\sqrt{A !}}\left[(2 \pi)^{3} \delta^{3}\left(\sum_{i=1}^{A} \boldsymbol{p}_{i}^{\star}\right)\right]^{1 / 2}\left|\left\{p^{\star}\right\}\right\rangle$

which agrees with the normalization in Eq. (A23).

\section{APPENDIX B: DERIVATION OF THE $\nu A \rightarrow \nu A$ CROSS SECTION}

\section{Scattering amplitude}

An effective SM Lagrangian in the four-fermion approximation

$$
\mathcal{L}(x)=\frac{G_{F}}{\sqrt{2}} L_{\mu}(x) H^{\mu}(x)
$$

should be accurate enough for the scattering of a low energy neutrino off of a nucleus. In Eq. (B1),

$$
L_{\mu}(x)=: \bar{\psi}_{\nu}(x) \gamma_{\mu}\left(1-\gamma_{5}\right) \psi_{\nu}(x):
$$

and 


$$
H^{\mu}(x)=\sum_{f=n, p}: \bar{\psi}_{f}(x) \gamma^{\mu}\left(g_{V}^{f}-g_{A}^{f} \gamma_{5}\right) \psi_{f}(x):
$$

are weak currents of neutrino and nucleons, respectively, written in the normal ordering represented by colons. The quantum fields $\psi_{\nu}(x)$ and $\psi_{n, p}(x)$ correspond to the neutrino and nucleons, respectively.

The $\mathbb{S}$-matrix amplitude $\left\langle P_{m}^{\prime}, k^{\prime}|\mathbb{S}| P_{n}, k\right\rangle$, to the first order of $G_{F}$, reads

$$
\begin{aligned}
\left\langle P_{m}^{\prime}, k^{\prime}|\mathbb{S}| P_{n}, k\right\rangle & =(2 \pi)^{4} \delta^{4}\left(q+P_{n}-P_{m}^{\prime}\right) i \mathcal{M}_{m n}, \\
i \mathcal{M}_{m n} & =i \frac{G_{F}}{\sqrt{2}} l_{\mu} H_{m n}^{\mu},
\end{aligned}
$$

where

$$
l_{\mu}\left(k, k^{\prime}\right)=\bar{u}\left(k^{\prime},-1\right) \gamma_{\mu}\left(1-\gamma_{5}\right) u(k,-1)
$$

and

$$
H_{m n}^{\mu}\left(P_{n}, P_{m}^{\prime}\right)=\left\langle P_{m}^{\prime}\left|H^{\mu}(0)\right| P_{n}\right\rangle .
$$

Using (A2), (B2) and the antisymmetric nature of the wave function, the hadronic current in (B5) can be found

$$
H_{m n}^{\mu}\left(P, P^{\prime}\right)=2 \sqrt{P_{n}^{0} P_{m}^{\prime 0}} h_{m n}^{\mu}
$$

with

$$
\begin{aligned}
h_{m n}^{\mu}= & \sum_{k=1}^{A} \int\left(\prod_{j=1}^{A} \frac{d \boldsymbol{p}_{j}^{\star}}{(2 \pi)^{3}}\right) \frac{\bar{u}\left(\boldsymbol{p}_{k}+\boldsymbol{q}, s_{k}\right) O_{k}^{\mu} u\left(\boldsymbol{p}_{k}, r_{k}\right)}{\sqrt{2 E_{\boldsymbol{p}_{k}} 2 E_{\boldsymbol{p}_{k}+\boldsymbol{q}}}} \\
& \times(2 \pi)^{3} \delta^{3}\left(\sum_{l=1}^{A} \boldsymbol{p}_{l}^{\star}\right) \tilde{\psi}_{m}^{*}\left(\left\{p_{\star}^{(k)}\right\}\right) \tilde{\psi}_{n}\left(\left\{p_{\star}\right\}\right),
\end{aligned}
$$

where

$$
\begin{aligned}
O_{k}^{\mu} & =\gamma^{\mu}\left(g_{V}^{k}-g_{A}^{k} \gamma_{5}\right) \\
& =\gamma^{\mu}\left(g_{L}^{k}\left(1-\gamma_{5}\right)+g_{R}^{k}\left(1+\gamma_{5}\right)\right)
\end{aligned}
$$

and the couplings $g_{V, A}^{k}$ are equal to $g_{V, A}^{p / n}$ when the index $k$ points to a proton/neutron. Left- and right-chirality couplings are expressed via vector $g_{V}^{p / n}$ and axial $g_{A}^{p / n}$ couplings as

$$
\begin{aligned}
& g_{L}^{p / n}=\frac{1}{2}\left(g_{V}^{p / n}+g_{A}^{p / n}\right), \\
& g_{R}^{p / n}=\frac{1}{2}\left(g_{V}^{p / n}-g_{A}^{p / n}\right) .
\end{aligned}
$$

In the SM, these couplings read

$$
\begin{aligned}
g_{V}^{p} & =\frac{1}{2}-2 \sin ^{2} \theta_{W}, & g_{A}^{p} & =\frac{1}{2}, \\
g_{L}^{p} & =\frac{1}{2}\left(1-2 \sin ^{2} \theta_{W}\right), & g_{R}^{p} & =-\sin ^{2} \theta_{W}, \\
g_{V}^{n} & =-\frac{1}{2}, & g_{A}^{n} & =-\frac{1}{2}, \\
g_{L}^{n} & =-1, & g_{R}^{n} & =0 .
\end{aligned}
$$

The arguments of $\tilde{\psi}_{m}^{*}\left(\left\{p_{\star}^{(k)}\right\}\right)$ and $\tilde{\psi}_{n}\left(\left\{p_{\star}\right\}\right)$ are $n$-tuples defined as $\left\{p_{\star}\right\}=\left(p_{1}^{\star} \ldots p_{A}^{\star}\right)$, where its $i$ th element, $p_{i}^{\star}=\left(\boldsymbol{p}_{i}^{\star}, r_{i}\right)$ and $\left\{p_{\star}^{(k)}\right\}$, is identical to $\left\{p_{\star}\right\}$ except for its $k$ th element, which reads as $\left(\boldsymbol{p}_{k}^{\star}+\boldsymbol{q}, s_{k}\right)$. The three momentum $\boldsymbol{p}_{k}$, used in the argument of the Dirac spinor $u$, is the $k$ th nucleon momentum in the laboratory frame given by (A20).

The hadronic current, corresponding to neutrino-nucleus scattering, is a sum of currents $\bar{u}\left(\boldsymbol{p}_{k}+\boldsymbol{q}, s_{k}\right) O_{k}^{\mu} u\left(\boldsymbol{p}_{k}, r_{k}\right)$ corresponding to the scattering of a neutrino off of the $k$ th nucleon with momentum in the laboratory $\boldsymbol{p}_{k}$ and spin projection $r_{k}$. The probability amplitude to find a nucleon in the $\left|P_{n}\right\rangle$ state of the nucleus with these quantum numbers is just the wave function $\tilde{\psi}_{n}\left(\left\{p_{\star}\right\}\right)$ in the momentum representation, which depends on momenta in the nucleus center-of-mass frame.

The outgoing nucleon has a three-momentum in the laboratory of $\boldsymbol{p}_{k}+\boldsymbol{q}$, and, in general, an arbitrary spin projection $s_{k}$. The corresponding probability amplitude to find a nucleon with exactly these quantum numbers is given similarly by the wave function $\tilde{\psi}_{m}^{*}\left(\left\{p_{\star}^{(k)}\right\}\right)$.

The denominator $\sqrt{2 E_{\boldsymbol{p}_{k}} 2 E_{\boldsymbol{p}_{k}+\boldsymbol{q}}}$ depends on the energies of the initial and final nucleons in the laboratory frame, and automatically accounts for the normalization of Dirac spinors $u^{\dagger}(\boldsymbol{p}, s) u(\boldsymbol{p}, s)=2 E_{\boldsymbol{p}}$.

The equal momenta of the initial and final state spectator nucleons are integrated out with the weight given by a product of initial and final state wave functions.

To proceed further, let us make the following simplifications. The current $\bar{u}\left(\boldsymbol{p}_{k}+\boldsymbol{q}, s_{k}\right) O_{k}^{\mu} u\left(\boldsymbol{p}_{k}, r_{k}\right)$ could be factorized out from the integral at an effective momentum $\boldsymbol{p}_{k}$ which we approximate to be given by a solution of Eq. (32). Also, we assume that the spin and momenta structures of $\tilde{\psi}_{n}$ could be factorized into a product $\tilde{\psi}_{n}$ and $\chi_{n}$

$$
\tilde{\psi}_{n}\left(\left\{p_{\star}\right\}\right)=\tilde{\psi}_{n}\left(\left\{\boldsymbol{p}_{\star}\right\}\right) \chi_{n}(\{r\}),
$$

which are functions of two $n$-tuples $\left\{\boldsymbol{p}_{\star}\right\}=\left(\boldsymbol{p}_{1}^{\star} \ldots \boldsymbol{p}_{A}^{\star}\right)$ and $\{r\}=\left(r_{1} \ldots r_{A}\right)$, respectively. The spin-functions can be normalized as follows

$$
\chi_{m}^{\dagger}(\{r\}) \chi_{n}(\{r\})=\delta_{n m} .
$$

Thus, Eq. (B7) can be rewritten as 


$$
\begin{aligned}
h_{m n}^{\mu}= & \sum_{k=1}^{A} \frac{\bar{u}\left(\boldsymbol{p}+\boldsymbol{q}, s_{k}\right) O_{k}^{\mu} u\left(\boldsymbol{p}, r_{k}\right)}{\sqrt{2 E_{\boldsymbol{p}} 2 E_{\boldsymbol{p}+\boldsymbol{q}}}} \chi_{m}^{*}\left(\left\{r^{(k)}\right\}\right) \chi_{n}(\{r\}) \\
& \times \int\left(\prod_{j=1}^{A} \frac{d \boldsymbol{p}_{j}}{(2 \pi)^{3}}\right)(2 \pi)^{3} \delta^{3}\left(\sum_{l=1}^{A} \boldsymbol{p}_{l}\right) \\
& \times \tilde{\psi}_{m}^{*}\left(\left\{\boldsymbol{p}_{\star}^{(k)}\right\}\right) \tilde{\psi}_{n}\left(\left\{\boldsymbol{p}_{\star}\right\}\right),
\end{aligned}
$$

where $\left\{r^{(k)}\right\}$ is an $n$-tuple identical to $\{r\}$, except its $k$ th element, which is equal to $s_{k}$.

A further insight could be gained by observing that one can rewrite the multidimensional integral in (B13) as the matrix element

$$
\begin{aligned}
& \int\left(\prod_{j=1}^{A} \frac{d \boldsymbol{p}_{j}^{\star}}{(2 \pi)^{3}}\right)(2 \pi)^{3} \delta^{3}\left(\sum_{l=1}^{A} \boldsymbol{p}_{l}^{\star}\right) \tilde{\psi}_{m}^{*}\left(\left\{\boldsymbol{p}_{\star}^{(k)}\right\}\right) \tilde{\psi}_{n}\left(\left\{\boldsymbol{p}_{\star}\right\}\right) \\
& =\left\langle m\left|e^{i \boldsymbol{q} \hat{\boldsymbol{X}}_{k}}\right| n\right\rangle \equiv f_{m n}^{k}(\boldsymbol{q}),
\end{aligned}
$$

where $\hat{\boldsymbol{X}}$ is the three-coordinate operator of the $k$ th nucleon.

Equation (B14) provides a clue in understanding the appearance of coherent and incoherent regimes in neutrinonucleus elastic and inelastic scattering.

A derivation of Eq. (B14) is facilitated if the following equality is observed

$$
\langle\boldsymbol{p}| e^{i q \hat{\boldsymbol{X}}}=\frac{\sqrt{2 E_{p}}}{\sqrt{2 E_{p+q}}}\langle\boldsymbol{p}+\boldsymbol{q}| .
$$

Equation (B15) can be proven with help of Eq. (A8). Using Eqs. (A24) and (B15), the matrix element $\left\langle m\left|e^{i q \hat{X}_{k}}\right| n\right\rangle$ can be calculated.

Therefore, combining Eqs. (B3), (B5), (B7), and (B14), one gets the matrix element of elastic neutrino-nucleus scattering

$$
\begin{aligned}
i \mathcal{M}_{m n}= & i \frac{G_{F}}{\sqrt{2}}\left(\frac{P_{n}^{0}}{E_{\boldsymbol{p}}} \frac{P_{m}^{\prime 0}}{E_{\boldsymbol{p}+\boldsymbol{q}}}\right)^{1 / 2} l_{\mu}\left(k, k^{\prime}\right) \sum_{k=1}^{A}\left\langle m\left|e^{i \boldsymbol{q} \hat{X}_{k}}\right| n\right\rangle \\
& \times \chi_{m}^{*}\left(\left\{r^{(k)}\right\}\right) \chi_{n}(\{r\}) \bar{u}\left(\boldsymbol{p}+\boldsymbol{q}, s_{k}\right) O_{k}^{\mu} u\left(\boldsymbol{p}, r_{k}\right) .
\end{aligned}
$$

We introduce the following notation for economy of space:

$$
\chi_{m}^{*}\left(\left\{r^{(k)}\right\}\right) \chi_{n}(\{r\}) \equiv \lambda^{m n}(s, r) .
$$

In general, the scattered nucleus may have a final spin state different with respect to the initial. We assume in what follows that initial and final states of the nucleus are eigenstates of the spin operator with quantum numbers $\left(J, J_{3}\right)$. One might observe that if $m=n$, then the amplitude $\lambda^{m n}(s, r)=\delta_{s r}$ for appropriate normalization of the spin wave function [see the normalization used in Eq. (B12)]. We denote for $m \neq n$ the corresponding amplitude as $\lambda_{s r}^{m n}$. Therefore, for any $m, n$

$$
\lambda^{m n}(s, r)=\delta_{m n} \delta_{s r}+\left(1-\delta_{m n}\right) \lambda_{s r}^{m n} .
$$

The multiplier in Eq. (B16) can be rewritten, factoring out the leading order term $m_{A} / m_{N}$ and the factor $C_{m n, 1}$ of the order of unity defined as

$$
C_{1, m n}^{1 / 2}=\left(\frac{P_{n}^{0}}{E_{\boldsymbol{p}}} \frac{P_{m}^{\prime 0}}{E_{\boldsymbol{p}+\boldsymbol{q}}}\right)^{1 / 2} \frac{m_{N}}{m_{A}} .
$$

Using Eqs. (B14), (B17) and (B19), one can represent Eq. (B16) as in Eq. (37).

\section{Cross sections}

The cross section corresponding to the matrix element in Eq. (B3) reads

$\frac{d^{2} \sigma_{m n}}{d E_{\nu}^{\prime} d \cos \theta}=\frac{-E_{\nu}^{\prime}\left|i \mathcal{M}_{m n}\right|^{2}}{2^{5} \pi E_{\nu}\left(m_{A}+\varepsilon_{n}\right)} \frac{\delta\left(E_{\nu}-E_{\nu}^{\prime}-T_{A}-\Delta \varepsilon_{m n}\right)}{m_{A}+T_{A}+\varepsilon_{m}}$,

where all kinematic variables are given in the laboratory frame in which the initial nucleus is assumed to be at rest, $E_{\nu}^{\prime}$ is given by Eq. (19) and $\Delta \varepsilon_{m n}=\varepsilon_{m}-\varepsilon_{n}$. The kinetic energy $T_{A}$ of the scattered nucleus is given by Eqs. (26) and (27). Integration over $E_{\nu}^{\prime}$ can be done with help of a Dirac $\delta$-function, providing energy conservation, thus yielding

$$
\begin{aligned}
\frac{d \sigma_{m n}}{d \cos \theta}= & \frac{-\left|i \mathcal{M}_{m n}\right|^{2}}{2^{5} \pi\left(m_{A}+\varepsilon_{n}\right)} \frac{E_{\nu}^{\prime}\left(m_{A}+T_{A}\right)}{E_{\nu}\left(m_{A}+T_{A}+\varepsilon_{m}\right)} \\
& \times \frac{1}{m_{A}+E_{\nu}(1-\cos \theta)-\Delta \varepsilon_{m n}},
\end{aligned}
$$

One can obtain $d \sigma_{m n} / d T_{A}$ using a very accurate approximation given in Eq. (27)

$$
\begin{aligned}
\frac{d \sigma_{m n}}{d T_{A}} & =\frac{d \sigma_{m n}}{d \cos \theta} \frac{d \cos \theta}{d T_{A}}=-\frac{d \sigma_{m n}}{d \cos \theta} \frac{m_{A}}{E_{\nu}\left(E_{\nu}-\Delta \varepsilon_{m n}\right)} \\
& =\frac{\left|i \mathcal{M}_{m n}\right|^{2}}{2^{5} \pi E_{\nu}^{2} m_{A}} C_{2, m n},
\end{aligned}
$$

where

$$
\begin{aligned}
C_{2, m n}= & \frac{E_{\nu}^{\prime}}{E_{\nu}-\Delta \varepsilon_{m n}} \frac{\left(1+\frac{T_{A}}{m_{A}}\right)\left(1+\frac{\varepsilon_{n}}{m_{A}}\right)^{-1}}{\left(1+\frac{T_{A}+\varepsilon_{m}}{m_{A}}\right)} \\
& \times\left(1+\frac{E_{\nu}(1-\cos \theta)-\Delta \varepsilon_{m n}}{m_{A}}\right)^{-1}
\end{aligned}
$$

is of the order of unity.

Combining Eqs. (37), (B14), and (B22), one gets an observable differential cross section defined in Eq. (42) 


$$
\begin{aligned}
\frac{d \sigma}{d T_{A}}= & \frac{G_{F}^{2} m_{A}}{2^{6} \pi m_{N}^{2} E_{\nu}^{2}} \\
& \times \sum_{k, j=1}^{A} \sum_{n} \omega_{n} C_{1, m n} C_{2, m n}\left(f_{n n}^{k} f_{n n}^{j *} \sum_{r}\left(l, h_{r r}^{k}\right) \sum_{s}\left(l, h_{s s}^{j}\right)^{\dagger}\right. \\
& \left.+\sum_{m \neq n} f_{m n}^{k} f_{m n}^{j *} \sum_{s r} \lambda_{s r}^{m n}\left(l, h_{s r}^{k}\right)\left(\sum_{s^{\prime} r^{\prime}} \lambda_{s^{\prime} r^{\prime}}^{m n}\left(l, h_{s^{\prime} r^{\prime}}^{j}\right)\right)^{\dagger}\right)
\end{aligned}
$$

expressed through the scalar products $\left(l, h_{s r}^{p / n}\right)$ of 4 -vectors with components $l^{\mu}\left(k, k^{\prime}\right)$ given by Eq. (B4) and

$$
\left(h_{s r}^{p / n}\right)_{\mu}=\bar{u}(\boldsymbol{p}+\boldsymbol{q}, s) O_{\mu}^{p / n} u(\boldsymbol{p}, r)
$$

where $\boldsymbol{p}$ is a solution of Eq. (32). In Eq. (B25), a superscript $p$ or $n$ appears when the index $k$ in $h_{s r}^{k}$ from Eq. (B24) points to a proton or to a neutron, respectively.

When an index $k$ or $j$ in Eq. (B24) points to a proton/ neutron, the form factors $f_{m n}^{k}$ should be read as $f_{m n}^{p / n}$, correspondingly.

Each of the $\left|\left(l, h^{p / n}\right)\right|^{2}$ terms given by Eq. (C12) and (C33) yields the common factor 64 $\left(s-m_{N}^{2}\right)^{2}$, where $s=$ $(p+k)^{2}$ is the total energy squared in the neutrino-nucleon center-of-mass frame, and $m_{N}$ is the mass of the nucleon. In the leading nonrelativistic approximation, this factor can be approximated as $2^{8} m_{N}^{2} E_{\nu}^{2}$. We denote a correction to this formula by a factor $C_{3, m n}$, accounting for the fact that the nucleon in the initial state has a nonzero three-momentum

$$
\left(s-m_{N}^{2}\right)^{2}=4 m_{N}^{2} E_{\nu}^{2} C_{3, m n} .
$$

In what follows, we denote by $g^{m n}$ the product of correction factors

$$
g^{m n}=C_{1, m n} C_{2, m n} C_{3, m n}
$$

which is of the order of unity.

Following our discussion of Eq. (37), we identify the second and third lines of Eq. (B24) as contributing to the coherent and incoherent cross sections. The factor $g^{m n}$ is, in general, different for coherent and incoherent terms. We take out these factors from the double summation at their effective values denoted by $g_{c}$ and $g_{i}$ for coherent and incoherent terms, respectively.

The summation over $n$ in the second line of Eq. (B24) leads to the form factors averaged over all initial states

$\sum_{n} \omega_{n} f_{n n}^{k} f_{n n}^{j *}= \begin{cases}\left|F_{p / n}(\boldsymbol{q})\right|^{2}, & (k, j) \rightarrow p p \quad \text { or } n n, \\ F_{p}(\boldsymbol{q}) F_{n}^{*}(\boldsymbol{q}), & (k, j) \rightarrow p n, \\ F_{n}(\boldsymbol{q}) F_{p}^{*}(\boldsymbol{q}), & (k, j) \rightarrow n p .\end{cases}$
Therefore, the second line of Eq. (B24) can be rewritten as

$$
\left|\sum_{k=1}^{Z} \sum_{r}\left(l, h_{r r}^{p}\right) F_{p}+\sum_{k=1}^{N} \sum_{r}\left(l, h_{r r}^{n}\right) F_{n}\right|^{2} .
$$

Let us work out the incoherent scattering encoded in the third line of Eq. (B24). A summation over $m, n$ cannot be done without a model for $\lambda_{s r}^{m n}$. If $\lambda_{s r}^{m n}$ would not depend on $m, n$ the corresponding summation could be performed as follows.

Consider the case when $k$ and $j$ point to the same type of the nucleon, e.g., to a proton.

If $k=j$, then

$$
\begin{aligned}
\sum_{n} \omega_{n} \sum_{m \neq n} f_{m n}^{k} f_{m n}^{k *}= & \sum_{n} \omega_{n}\left[\sum_{m} f_{m n}^{k} f_{m n}^{k *}-f_{n n}^{k} f_{n n}^{k *}\right] \\
= & \sum_{n} \omega_{n}\left[\left\langle n\left|e^{-i \boldsymbol{q} \boldsymbol{X}_{k}} \sum_{m}\right| m\right\rangle\left\langle m\left|e^{i \boldsymbol{q} \boldsymbol{X}_{k}}\right| n\right\rangle\right] \\
& -\left|F_{p}(\boldsymbol{q})\right|^{2} \\
= & 1-\left|F_{p}(\boldsymbol{q})\right|^{2},
\end{aligned}
$$

accounting for the equality $\sum_{m}|m\rangle\langle m|=\hat{I}$, using Eq. (B28) and normalizations in Eq. (A23) and $\sum_{n} \omega_{n}=1$.

If $k \neq j$, then following a consideration similar to Eq. (B30) one may find that

$$
\sum_{n} \omega_{n} \sum_{m \neq n} f_{m n}^{k} f_{m n}^{j *}=\left\langle\operatorname{cov}\left(e^{-i \boldsymbol{q} \hat{X}_{j}}, e^{i q \hat{X}_{k}}\right)\right\rangle_{p}
$$

where the right-hand-side of Eq. (B31) is a covariance of quantum operators $e^{-i q \hat{X}_{j}}$ and $e^{i q \hat{X}_{k}}$ on $|n\rangle$, whose state reads

$$
\begin{aligned}
& \operatorname{cov}_{n n}\left(e^{-i q \hat{X}_{j}}, e^{i q \hat{X}_{k}}\right) \\
& \quad=\left\langle n\left|e^{-i q \hat{X}_{j}} e^{i q \hat{X}_{k}}\right| n\right\rangle-\left\langle n\left|e^{i q \hat{X}_{k}}\right| n\right\rangle\left\langle n\left|e^{-i q \hat{X}_{j}}\right| n\right\rangle .
\end{aligned}
$$

The subscript $p$ in Eq. (B31) refers to a proton.

The averaging $\langle\ldots\rangle$ in Eq. (B31) is given by

$$
\left\langle\operatorname{cov}\left(e^{-i \boldsymbol{q} \hat{X}_{j}}, e^{i q \hat{X}_{k}}\right)\right\rangle_{p}=\sum_{n} \omega_{n} \operatorname{cov}_{n n}\left(e^{-i q \hat{X}_{j}}, e^{i \boldsymbol{q} \hat{X}_{k}}\right) .
$$

At both, $\boldsymbol{q} \rightarrow 0$ and $\boldsymbol{q} \rightarrow \infty$

$$
\begin{aligned}
\lim _{\boldsymbol{q} \rightarrow 0}\left\langle\operatorname{cov}\left(e^{-i \boldsymbol{q} \hat{X}_{j}}, e^{i \boldsymbol{q} \hat{X}_{k}}\right)\right\rangle_{p} & =0, \\
\lim _{\boldsymbol{q} \rightarrow \infty}\left\langle\operatorname{cov}\left(e^{-i q \hat{X}_{j}}, e^{i \boldsymbol{q} \hat{X}_{k}}\right)\right\rangle_{p} & =0 .
\end{aligned}
$$

In the case of weak correlations of nucleons in a nucleus, the covariances, like in Eq. (B31) vanish. For example, in models like the nuclear shell model, where a multiparticle wave function is constructed in terms of a product 
of one-particle wave functions, the covariance in Eq. (B31) is identically zero.

The smallness of the covariance in Eq. (B31) is the reason why the inelastic cross section is, to good accuracy, linearly dependent on the number of nucleons. In what follows, the covariance terms are neglected.

The same considerations apply to the scattering on a neutron. It is straightforward to show that in the case of mixing neutron and proton amplitudes one gets (let $k$ point to a proton and $j$ point to a neutron, and now automatically $k \neq j$ )

$$
\sum_{n} \omega_{n} \sum_{m \neq n} f_{m n}^{k} f_{m n}^{j *}=\left\langle\operatorname{cov}\left(e^{i q \hat{X}_{k}}, e^{-i q \hat{X}_{j}}\right)\right\rangle_{p n}
$$

which can also be neglected.

As mentioned above the exact summation should consider the spin amplitude $\lambda_{s r}^{m n}$. We approximate the summation by replacing $\lambda_{s r}^{m n}$ by its average value $\lambda_{s r}^{p / n}$ for protons and neutrons, respectively.

Therefore, the third line of Eq. (B24) reads

$$
\begin{aligned}
& \sum_{k=1}^{Z} \sum_{s r}\left|\lambda_{s r}^{p}\right|^{2}\left|\left(l, h_{s r}^{p}\right)\right|^{2}\left(1-\left|F_{p}\right|^{2}\right) \\
& \quad+\sum_{k=1}^{N} \sum_{s r}\left|\lambda_{s r}^{n}\right|^{2}\left|\left(l, h_{s r}^{n}\right)\right|^{2}\left(1-\left|F_{n}\right|^{2}\right) .
\end{aligned}
$$

Combining Eqs. (B24), (B29), and (B36), one gets the differential cross section in Eq. (44).

\section{APPENDIX C: CALCULATION OF THE SCALAR PRODUCT $(l, h)$}

The third line of Eq. (44) prompts us to calculate the scalar product of two currents $\bar{u}\left(k^{\prime}\right) O^{\mu} u(k) \cdot \bar{u}\left(p^{\prime}\right) O_{\mu}^{\prime} u(p)$, where $O_{\mu}, O_{\mu}^{\prime}$ are Dirac matrices. The use of a standard powerful technique, which consists of the calculation of traces of Dirac $\gamma$-matrices, is not helpful for this problem. This is because all four momenta $k, k^{\prime}, p$ and $p^{\prime}$ are different and one cannot use the well-known formula for Dirac spinors

$$
u(\boldsymbol{p}, r) \bar{u}(\boldsymbol{p}, r)=\frac{1}{2}(\not p+m)\left(1+\gamma_{5} \phi_{r}\right),
$$

where $s_{r}$ is four-vector of the fermion spin.

To simplify intermediate formulas, we calculate the scalar product of the neutrino and nucleon currents in their center-of-mass frame, where energies of incoming and outgoing fermions are equal. In what follows, in this section all quantities depending on kinematic variables are given in the neutrino-nucleon center-of-mass frame.

Energies $E_{\nu}$ and $E_{N}$, of the neutrino and nucleon, respectively, read

$$
E_{\nu}=\frac{s-m^{2}}{2 \sqrt{s}}, \quad E_{N}=\frac{s+m^{2}}{2 \sqrt{s}}
$$

where $s=(p+k)^{2}$ and $m$ gives the mass of the nucleon.

In the Dirac basis, the spinor of a nucleon with threemomentum $\boldsymbol{p}$ and index $r= \pm 1$ reads

$$
u(\boldsymbol{p}, r)=\left(\begin{array}{c}
\lambda_{+} \\
\lambda_{-} \alpha_{p}
\end{array}\right) \chi_{r}(\boldsymbol{p}),
$$

where $\lambda_{ \pm}=\sqrt{E_{N} \pm m}$ and $\alpha_{\boldsymbol{p}}=\boldsymbol{n}_{\boldsymbol{p}} \cdot \boldsymbol{\sigma}$, in which $\boldsymbol{n}_{\boldsymbol{p}}$ is a unit vector along $\boldsymbol{p}$, and $\boldsymbol{\sigma}=\left(\sigma_{1}, \sigma_{2}, \sigma_{3}\right)$ is a three-vector of Pauli matrices. The index $r$ enumerates two linearly independent two-spinors $\chi_{r}(\boldsymbol{p})$.

The vector and axial currents of the nucleon read

$$
\begin{aligned}
\bar{u}\left(\boldsymbol{p}^{\prime}, r^{\prime}\right) \gamma^{\mu} u(\boldsymbol{p}, r) & \equiv V_{r^{\prime} r}^{\mu}\left(\boldsymbol{p}^{\prime}, \boldsymbol{p}\right), \\
\bar{u}\left(\boldsymbol{p}^{\prime}, r^{\prime}\right) \gamma^{\mu} \gamma^{5} u(\boldsymbol{p}, r) & \equiv A_{r^{\prime} r}^{\mu}\left(\boldsymbol{p}^{\prime}, \boldsymbol{p}\right),
\end{aligned}
$$

where

$$
\begin{aligned}
& V_{r^{\prime} r}^{0}\left(\boldsymbol{p}^{\prime}, \boldsymbol{p}\right)=\chi_{r^{\prime}}^{\dagger}\left(\boldsymbol{n}_{\boldsymbol{p}^{\prime}}\right)\left[\lambda_{+}^{2}+\lambda_{-}^{2} \alpha_{p^{\prime}} \alpha_{\boldsymbol{p}}\right] \chi_{r}\left(\boldsymbol{n}_{\boldsymbol{p}}\right), \\
& \boldsymbol{V}_{r^{\prime} r}\left(\boldsymbol{p}^{\prime}, \boldsymbol{p}\right)=\chi_{r^{\prime}}^{\dagger}\left(\boldsymbol{n}_{\boldsymbol{p}^{\prime}}\right) \lambda_{+} \lambda_{-}\left[\boldsymbol{\sigma} \alpha_{\boldsymbol{p}}+\alpha_{p^{\prime}} \boldsymbol{\sigma}\right] \chi_{r}\left(\boldsymbol{n}_{\boldsymbol{p}}\right), \\
& A_{r^{\prime} r}^{0}\left(\boldsymbol{p}^{\prime}, \boldsymbol{p}\right)=\chi_{r^{\prime}}^{\dagger}\left(\boldsymbol{n}_{\boldsymbol{p}^{\prime}}\right) \lambda_{+} \lambda_{-}\left[\alpha_{p^{\prime}}+\alpha_{\boldsymbol{p}}\right] \chi_{r}\left(\boldsymbol{n}_{\boldsymbol{p}}\right), \\
& \boldsymbol{A}_{r^{\prime} r}\left(\boldsymbol{p}^{\prime}, \boldsymbol{p}\right)=\chi_{r^{\prime}}^{\dagger}\left(\boldsymbol{n}_{\boldsymbol{p}^{\prime}}\right)\left[\lambda_{+}^{2} \boldsymbol{\sigma}+\lambda_{-}^{2} \alpha_{p^{\prime}} \boldsymbol{\sigma} \alpha_{\boldsymbol{p}}\right] \chi_{r}\left(\boldsymbol{n}_{\boldsymbol{p}}\right) .
\end{aligned}
$$

The neutrino spinor, vector, and axial currents read analogously to Eq. (C2)-(C4) with the replacement $\lambda_{ \pm} \rightarrow \sqrt{E_{\nu}}$.

Unit vectors along three-momenta of the incoming and outgoing neutrino and nucleon are defined as

$$
\begin{aligned}
\boldsymbol{n}_{\boldsymbol{k}} & =(0,0,1), \quad \boldsymbol{n}_{\boldsymbol{p}}=-\boldsymbol{n}_{\boldsymbol{k}}, \\
\boldsymbol{n}_{\boldsymbol{k}^{\prime}} & =(\cos \varphi \sin \theta, \sin \varphi \sin \theta, \cos \theta), \quad \boldsymbol{n}_{\boldsymbol{p}^{\prime}}=-\boldsymbol{n}_{\boldsymbol{k}^{\prime}} .
\end{aligned}
$$

It is convenient to specify a basis of two-component spinors $\chi_{r}$ to perform the calculations in Eq. (C3). Summation over $r, r^{\prime}$ in the incoherent term of Eq. (44) are simpler in the helicity basis in which $r, r^{\prime}$ are helicity eigenvalues. The coherent term of Eq. (44) requires consideration of the nucleon current with conservation of spin projection on the given axis. For this purpose, a basis of $\chi_{r}$ twospinors, which are eigenstates of the $\sigma_{3}=\left(\boldsymbol{n}_{\boldsymbol{k}} \cdot \boldsymbol{\sigma}\right)$ matrix, is more appropriate. It is apparent that the physical observable does not depend on the basis chosen.

\section{Helicity basis}

In the helicity basis, the two-spinor $\chi_{r}(\boldsymbol{p})$ is an eigenvector of the helicity operator $\boldsymbol{n}_{p} \cdot \boldsymbol{\sigma}$

$$
\boldsymbol{n}_{\boldsymbol{p}} \cdot \boldsymbol{\sigma} \chi_{r}\left(\boldsymbol{n}_{\boldsymbol{p}}\right)=r \chi_{r}\left(\boldsymbol{n}_{\boldsymbol{p}}\right)
$$

with an eigenvalue $r= \pm 1$, known as the helicity or doubled spin projection on a particle's three-momentum. 
Two-component normalized to unity spinors $\chi$ corresponding to the incoming and outgoing neutrino and nucleon with definite helicities in their center-of-mass frame, can be read

$$
\begin{array}{ll}
\chi_{+}\left(\boldsymbol{n}_{\boldsymbol{k}}\right)=\left(\begin{array}{l}
1 \\
0
\end{array}\right), & \chi_{+}\left(\boldsymbol{n}_{\boldsymbol{k}^{\prime}}\right)=\left(\begin{array}{c}
\cos \frac{\theta}{2} \\
e^{i \varphi} \sin \frac{\theta}{2}
\end{array}\right), \\
\chi_{-}\left(\boldsymbol{n}_{\boldsymbol{k}}\right)=\left(\begin{array}{c}
0 \\
1
\end{array}\right), & \chi_{-}\left(\boldsymbol{n}_{\boldsymbol{k}^{\prime}}\right)=\left(\begin{array}{c}
-e^{-i \varphi} \sin \frac{\theta}{2} \\
\cos \frac{\theta}{2}
\end{array}\right), \\
\chi_{+}\left(\boldsymbol{n}_{\boldsymbol{p}}\right)=\left(\begin{array}{c}
0 \\
-1
\end{array}\right), & \chi_{+}\left(\boldsymbol{n}_{\boldsymbol{p}^{\prime}}\right)=\left(\begin{array}{c}
\sin \frac{\theta}{2} \\
-e^{i \varphi} \cos \frac{\theta}{2}
\end{array}\right), \\
\chi_{-}\left(\boldsymbol{n}_{\boldsymbol{p}}\right)=\left(\begin{array}{c}
1 \\
0
\end{array}\right), & \chi_{-}\left(\boldsymbol{n}_{\boldsymbol{p}^{\prime}}\right)=\left(\begin{array}{c}
e^{-i \varphi} \cos \frac{\theta}{2} \\
\sin \frac{\theta}{2}
\end{array}\right) .
\end{array}
$$

Let us display explicitly the vector and axial currents in the helicity basis from Eqs. (C3) and (C4) for the nucleon, denoting the basis by the $\chi$ superscript

$$
\begin{aligned}
& V_{++}^{\chi}=2\left(c_{\theta / 2} e^{-i \varphi} E_{N},\left(-s_{\theta / 2}, i s_{\theta / 2},-c_{\theta / 2} e^{-i \varphi}\right) E_{\nu}\right), \\
& V_{--}^{\chi}=2\left(c_{\theta / 2} e^{+i \varphi} E_{N},\left(-s_{\theta / 2},-i s_{\theta / 2},-c_{\theta / 2} e^{+i \varphi}\right) E_{\nu}\right), \\
& V_{+-}^{\chi}=2 m\left(s_{\theta / 2}, 0,0,0\right), \\
& V_{-+}^{\chi}=2 m\left(-s_{\theta / 2}, 0,0,0\right), \\
& A_{++}^{\chi}=2\left(c_{\theta / 2} e^{-i \varphi} E_{\nu},\left(-s_{\theta / 2}, i s_{\theta / 2},-c_{\theta / 2} e^{-i \varphi}\right) E_{N}\right), \\
& A_{--}^{\chi}=2\left(-c_{\theta / 2} e^{+i \varphi} E_{\nu},\left(s_{\theta / 2}, i s_{\theta / 2}, c_{\theta / 2} e^{+i \varphi}\right) E_{N}\right), \\
& A_{+-}^{\chi}=2 m\left(0,-c_{\theta / 2} e^{-i \varphi},-i c_{\theta / 2} e^{-i \varphi}, s_{\theta / 2}\right), \\
& A_{-+}^{\chi}=2 m\left(0,-c_{\theta / 2} e^{+i \varphi}, i c_{\theta / 2} e^{+i \varphi}, s_{\theta / 2}\right)
\end{aligned}
$$

and neutrino

$$
\begin{aligned}
& V_{-_{-}}^{\chi}=2 E_{\nu}\left(c_{\theta / 2}, s_{\theta / 2} e^{+i \varphi},-i s_{\theta / 2} e^{+i \varphi}, c_{\theta / 2}\right), \\
& A_{--}^{\chi}=2 E_{\nu}\left(-c_{\theta / 2},-s_{\theta / 2} e^{+i \varphi}, i s_{\theta / 2} e^{+i \varphi},-c_{\theta / 2}\right),
\end{aligned}
$$

where for the sake of compactness

$$
c_{\theta / 2} \equiv \cos \frac{\theta}{2}, \quad s_{\theta / 2} \equiv \sin \frac{\theta}{2} .
$$

For a neutrino, assuming its vanishing mass,

$$
V_{+-}=V_{-+}=A_{+-}=A_{-+}=0,
$$

manifesting neutrino helicity conservation in weak interactions. In Eq. (C9), only the left-handed neutrino currents required to calculate the elastic neutrino-nucleus cross section are shown.

Now it is straightforward to calculate the scalar product $\left(l, h_{r^{\prime} r}\right)$ equal to $\bar{u}\left(k^{\prime},-1\right) \gamma^{\mu}\left(1-\gamma^{5}\right) u(k,-1) \cdot \bar{u}\left(p^{\prime}, r^{\prime}\right) \gamma_{\mu}\left(g_{V}-g_{A} \gamma^{5}\right) u(p, r)$

using Eqs. (C8) and (C9)

$$
\begin{aligned}
& \left(l, h_{++}^{\chi}\right)=8\left(s-m^{2}\right) e^{-i \varphi} \cos ^{2} \frac{\theta}{2} g_{R}, \\
& \left(l, h_{--}^{\chi}\right)=8\left(s-m^{2}\right) e^{+i \varphi}\left(g_{L}-g_{R} \sin ^{2} \frac{\theta}{2} \frac{m^{2}}{s}\right), \\
& \left(l, h_{+-}^{\chi}\right)=8\left(s-m^{2}\right) \frac{m}{\sqrt{s}} \sin \frac{\theta}{2} \cos \frac{\theta}{2} g_{R}, \\
& \left(l, h_{-+}^{\chi}\right)=-8\left(s-m^{2}\right) \frac{m}{\sqrt{s}} \sin \frac{\theta}{2} \cos \frac{\theta}{2} g_{R},
\end{aligned}
$$

where $g_{L / R}$ are left- and right-handed chirality weak couplings of the nucleon defined in Eq. (B9).

Using Eq. (C12) and the relationship between Bjorken $y$ and $\sin ^{2} \frac{\theta}{2}$ in the neutrino-nucleon center-ofmass frame

$$
y=\frac{(p, q)}{(p, k)}, \quad \sin ^{2} \frac{\theta}{2}=\frac{y s}{s-m^{2}}
$$

one can verify that a well known result determining the cross section of the neutrino-nucleon scattering with $Z^{0}$ boson exchange is reproduced

$$
\begin{aligned}
& \sum_{r, r^{\prime}}\left|\left(l, h_{r^{\prime} r}^{\chi}\right)\right|^{2} \\
& \quad=2^{6}\left(s-m^{2}\right)^{2}\left(g_{L}^{2}+g_{R}^{2}(1-y)^{2}-2 g_{L} g_{R} \frac{y m^{2}}{s-m^{2}}\right) .
\end{aligned}
$$

\section{2. $\sigma_{3}$ basis}

We quantize the nucleon's spin along the incoming neutrino direction $\boldsymbol{n}_{\boldsymbol{k}}$ in the neutrino-nucleon center-ofmass frame. This implies that the corresponding twospinor, which we denote now by $\eta_{r}$, is an eigenvector of the $\boldsymbol{n}_{\boldsymbol{k}} \cdot \boldsymbol{\sigma}=\sigma_{3}$ matrix

$$
\sigma_{3} \eta_{r}=r \eta_{r}
$$

These two-spinors read

$$
\eta_{+}=\left(\begin{array}{l}
1 \\
0
\end{array}\right), \quad \eta_{-}=\left(\begin{array}{l}
0 \\
1
\end{array}\right) .
$$

Using these two-spinors instead of $\chi_{ \pm}$in Eq. (C3) and (C4), the nucleon's vector and axial currents, denoting the basis in (C16) by the superscript $\eta$, read 


$$
\begin{aligned}
& V_{++}^{\eta}=\left(E_{N}+m+\cos \theta\left(E_{N}-m\right),-\sin \theta e^{-i \varphi} E_{\nu},-i \sin \theta e^{-i \varphi} E_{\nu},-(1+\cos \theta) E_{\nu}\right), \\
& V_{--}^{\eta}=\left(E_{N}+m+\cos \theta\left(E_{N}-m\right),-\sin \theta e^{+i \varphi} E_{\nu}, i \sin \theta e^{+i \varphi} E_{\nu},-(1+\cos \theta) E_{\nu}\right), \\
& V_{+-}^{\eta}=\left(-\sin \theta e^{-i \varphi}\left(E_{N}-m\right),(1-\cos \theta) E_{\nu},-i(1-\cos \theta) E_{\nu}, \sin \theta e^{-i \varphi} E_{\nu}\right), \\
& V_{-+}^{\eta}=\left(+\sin \theta e^{+i \varphi}\left(E_{N}-m\right),-(1-\cos \theta) E_{\nu},-i(1-\cos \theta) E_{\nu},-\sin \theta e^{+i \varphi} E_{\nu}\right), \\
& A_{++}^{\eta}=\left(-(1+\cos \theta) E_{\nu}, \sin \theta e^{-i \varphi}\left(E_{N}-m\right), i \sin \theta e^{-i \varphi}\left(E_{N}-m\right), E_{N}+m+\cos \theta\left(E_{N}-m\right)\right), \\
& A_{--}^{\eta}=\left((1+\cos \theta) E_{\nu},-\sin \theta e^{+i \varphi}\left(E_{N}-m\right), i \sin \theta e^{+i \varphi}\left(E_{N}-m\right),-\left(E_{N}+m+\cos \theta\left(E_{N}-m\right)\right)\right), \\
& A_{+-}^{\eta}=\left(-\sin \theta e^{-i \varphi} E_{\nu}, E_{N}+m-\cos \theta\left(E_{N}-m\right),-i\left(E_{N}+m-\cos \theta\left(E_{N}-m\right)\right), \sin \theta e^{-i \varphi}\left(E_{N}-m\right)\right), \\
& A_{-+}^{\eta}=\left(-\sin \theta e^{+i \varphi} E_{\nu}, E_{N}+m-\cos \theta\left(E_{N}-m\right), i\left(E_{N}+m-\cos \theta\left(E_{N}-m\right)\right), \sin \theta e^{+i \varphi}\left(E_{N}-m\right)\right) .
\end{aligned}
$$

The vector and axial currents calculated in Eq. (C8) and in Eq. (C17) differ because the mathematical and physical sense of $r, r^{\prime}$ eigenvalues are different. In Eq. (C8), $r, r^{\prime}$ are projections of the nucleon spin onto incoming and outgoing momenta of the nucleon, while in Eq. (C17) these are projections onto a fixed axis (chosen to be along the incoming neutrino three-momentum). One possible illustration is an example of the nucleon scattered backward $(\cos \theta=-1)$. In this case, $(++)$ in the helicity basis represents the nucleon's spin-flip, while in the basis with fixed quantization axis [as defined in Eq. (C16)], the nucleon's spin does not change its orientation.

Let us briefly review the results obtained in Eq. (C17) to gain further insight. For this purpose, we consider three cases most relevant for this paper: (i) the nucleon forward scattering $(\cos \theta=1)$, (ii) the nucleon backward scattering $(\cos \theta=-1)$ and (iii) the nonrelativistic regime $(\sqrt{s} \rightarrow m)$.

(i) The vector currents

$$
\lim _{\cos \theta \rightarrow 1} V_{++}^{\eta}=\lim _{\cos \theta \rightarrow 1} V_{--}^{\eta}=2\left(E_{N}, 0,0,-\left|\boldsymbol{P}_{N}\right|\right)
$$

reduce to the 4-momentum of the nucleon moving towards the incoming neutrino. The spin-flip in the vector currents are impossible

$$
\lim _{\cos \theta \rightarrow 1} V_{+-}^{\eta}=\lim _{\cos \theta \rightarrow 1} V_{-+}^{\eta}=0 .
$$

On the contrary, the axial current makes the spin-flip possible even for the forward scattering generating nonzero components of the axial current in the transverse plane

$$
\begin{aligned}
& \lim _{\cos \theta \rightarrow 1} A_{+-}^{\eta}=2 m(0,1,-i, 0), \\
& \lim _{\cos \theta \rightarrow 1} A_{-+}^{\eta}=2 m(0,1, i, 0) .
\end{aligned}
$$

In this limit, there is an exact cancellation of the sum of axial currents with opposite spins

$$
\lim _{\cos \theta \rightarrow 1}\left(A_{++}^{\eta}+A_{--}^{\eta}\right)=0 .
$$

This cancellation can be understood by recalling that the axial current of the fermion with the same initial and final momenta $p$ and same spin projection $r$ is proportional to the 4-spin vector $s^{\mu}$

$$
\bar{u}(p, r) \gamma^{\mu} \gamma^{5} u(p, r)=2 m r s^{\mu} .
$$

Therefore,

$\bar{u}(p,+1) \gamma^{\mu} \gamma^{5} u(p,+1)+\bar{u}(p,-1) \gamma^{\mu} \gamma^{5} u(p,-1)=0$,

which exactly corresponds to Eq. (C21) for the forward scattering of the nucleon.

(ii) The vector currents conserving spin projection reduce to

$$
\lim _{\cos \theta \rightarrow-1} V_{++}^{\eta}=\lim _{\cos \theta \rightarrow-1} V_{--}^{\eta}=2 m(1,0,0,0) .
$$

The spin-flip is also possible

$$
\begin{aligned}
& \lim _{\cos \theta \rightarrow-1} V_{+-}^{\eta}=2\left|\boldsymbol{P}_{N}\right|(0,1,-i, 0), \\
& \lim _{\cos \theta \rightarrow-1} V_{-+}^{\eta}=2\left|\boldsymbol{P}_{N}\right|(0,-1,-i, 0)
\end{aligned}
$$

generating nonzero components of the vector current in the transverse plane. The axial currents conserving spin projection reduce to

$$
\begin{aligned}
& \lim _{\cos \theta \rightarrow-1} A_{++}^{\eta}=2 m(0,0,0,1), \\
& \lim _{\cos \theta \rightarrow-1} A_{--}^{\eta}=2 m(0,0,0,-1) .
\end{aligned}
$$


The spin-flip is also possible

$$
\begin{aligned}
& \lim _{\cos \theta \rightarrow-1} A_{+-}^{\eta}=2 E_{N}(0,1,-i, 0), \\
& \lim _{\cos \theta \rightarrow-1} A_{-+}^{\eta}=2 E_{N}(0,1, i, 0) .
\end{aligned}
$$

(iii) The vector current conserves the spin projection

$$
\begin{aligned}
& \lim _{\sqrt{s} \rightarrow m} V_{++}^{\eta}=\lim _{\sqrt{s} \rightarrow m} V_{--}^{\eta}=2 m(1,0,0,0), \\
& \lim _{\sqrt{s} \rightarrow m} V_{+-}^{\eta}=V_{-+}^{\eta}=0 .
\end{aligned}
$$

The axial current is nonzero for both cases: spin projection conserved

$$
\begin{aligned}
& \lim _{\sqrt{s} \rightarrow m} A_{++}^{\eta}=2 m(0,0,0,1), \\
& \lim _{\sqrt{s} \rightarrow m} A_{--}^{\eta}=2 m(0,0,0,-1)
\end{aligned}
$$

and when the spin-flip occurred

$$
\begin{aligned}
& \lim _{\sqrt{s} \rightarrow m} A_{+-}^{\eta}=2 m(0,1,-i, 0), \\
& \lim _{\sqrt{s} \rightarrow m} A_{-+}^{\eta}=2 m(0,1, i, 0) .
\end{aligned}
$$

One might observe, that in this limit, similar to Eq. (C21),

$$
\lim _{\sqrt{s} \rightarrow m}\left(A_{++}^{\eta}+A_{--}^{\eta}\right)=0
$$

which implies that in the coherent term of Eq. (44) there is a cancellation of the axial currents for spinless nuclei. A more accurate statement can be drawn considering the exact formula in Eq. (C17)

$$
\begin{aligned}
A_{++}^{\eta}+A_{--}^{\eta} & =-2 i \sin \theta\left(E_{N}-m\right)(0, \sin \varphi, \cos \varphi, 0) \\
& \simeq-i \frac{k_{0}^{2}}{m} \sin \theta(0, \sin \varphi, \cos \varphi, 0) \quad(\mathrm{C} 32)
\end{aligned}
$$

In general, this four-vector is nonzero unless the neutrino energy $k_{0}$ in the laboratory frame is not zero and the scattering angle $\theta \neq 0$ or $\pi$.

Once the vector and axial currents of the nucleon are calculated, it is straightforward to calculate the scalar product $\left(l, h_{r^{\prime} r}\right)$ in analogy to the results obtained in the helicity basis Eq. (C12) using Eqs. (C9) and (C17)

$$
\begin{aligned}
\left(l, h_{++}^{\eta}\right) & =8\left(s-m^{2}\right) \cos \frac{\theta}{2}\left(g_{L}-g_{R} \sin ^{2} \frac{\theta}{2} \frac{m}{\sqrt{s}}\left(1-\frac{m}{\sqrt{s}}\right)\right), \\
\left(l, h_{--}^{\eta}\right) & =8\left(s-m^{2}\right) \cos \frac{\theta}{2}\left(1-\sin ^{2} \frac{\theta}{2}\left(1-\frac{m}{\sqrt{s}}\right)\right) g_{R}, \\
\left(l, h_{+-}^{\eta}\right) & =-8\left(s-m^{2}\right) e^{-i \varphi} \sin \frac{\theta}{2} \cos ^{2} \frac{\theta}{2}\left(1-\frac{m}{\sqrt{s}}\right) g_{R}, \\
\left(l, h_{-+}^{\eta}\right) & =8\left(s-m^{2}\right) e^{i \varphi} \sin \frac{\theta}{2} \\
& \left.\times\left(g_{L}-g_{R} \frac{m}{\sqrt{s}}\left(1-\sin ^{2} \frac{\theta}{2}\left(1-\frac{m}{\sqrt{s}}\right)\right)\right), \quad \text { C } 333\right)
\end{aligned}
$$

The scalar products $\left(l, h_{r r}^{\eta}\right)$ in Eq. (C33) differ from those in Eq. (C12).

(i) The $\varphi$-dependence magically disappeared in the first two lines of Eq. (C33) which determine the coherent cross section. In $\left(l, h_{++}^{\eta}\right)$, it happened because the exponent $e^{i \varphi}$ of the neutrino current in Eq. (C9) cancels the exponent $e^{-i \varphi}$ of nucleon currents $V_{++}$ and $A_{++}$in Eq. (C17). In $\left(l, h_{++}^{\eta}\right)$, it happened because the corresponding terms, depending now on $e^{i 2 \varphi}$, cancel each other due to the difference in their relative signs.

The scalars $\left(l, h_{+-}^{\eta}\right)$ and $\left(l, h_{-+}^{\eta}\right)$ have a $\varphi$-dependence. However this dependence can not be observed because these terms do not contribute to the coherent cross section where they could be interfering.

(ii) For the forward scattering $\theta \rightarrow 0$, the sum of the first two lines in Eq. (C33) does not depend on $g_{A}$

$$
\lim _{y \rightarrow 0}\left(\left(l, h_{++}^{\eta}\right)+\left(l, h_{--}^{\eta}\right)\right)=8\left(s-m^{2}\right) g_{V} .
$$

Two other currents with spin-flip vanish in this limit.

(iii) Both scalar products $\left(l, h_{r r}^{\eta}\right)$ vanish if the neutrino scatters backward $(\theta=\pi)$ in their center-of-mass frame because it corresponds to a change of the total spin of the neutrino-nucleon system by one unit. This result explains why the coherent cross section vanishes when the recoil nucleus has the maximum kinetic energy.

(iv) In the nonrelativistic limit,

$$
\begin{aligned}
& \lim _{\sqrt{s} \rightarrow m} \frac{\left(l, h_{++}^{\eta}\right)}{8\left(s-m^{2}\right)}=\cos \frac{\theta}{2} g_{L}, \\
& \lim _{\sqrt{s} \rightarrow m} \frac{\left(l, h_{--}^{\eta}\right)}{8\left(s-m^{2}\right)}=\cos \frac{\theta}{2} g_{R}, \\
& \lim _{\sqrt{s} \rightarrow m} \frac{\left(l, h_{+-}^{\eta}\right)}{8\left(s-m^{2}\right)}=0, \\
& \lim _{\sqrt{s} \rightarrow m} \frac{\left(l, h_{-+}^{\eta}\right)}{8\left(s-m^{2}\right)}=e^{i \varphi} \sin \frac{\theta}{2} g_{A},
\end{aligned}
$$

where we kept the $8\left(s-m^{2}\right)$ factor in the denominators because it is precisely canceled in the calculation of the corresponding cross section. 
It is interesting to observe two distinct limits of the last two lines in Eq. (C35) for similar spin-flipped configurations. In the nonrelativistic limit, the vector currents with spin-flip vanish as can be seen in Eq. (C28) and only the transverse components of the axial currents in Eq. (C30) survive. However, the axial current $A_{+-}^{\eta}$ in Eq. (C30) turned out to be orthogonal to the neutrino current at $\sqrt{s} \rightarrow m$. At the same time, the scalar product of the latter and of $A_{-+}^{\eta}$ from Eq. (C30) is nonzero and proportional to the axial coupling $g_{A}$.

\section{Relation between helicity and $\sigma_{3}$ bases}

The eigenvectors $\chi_{ \pm}$and $\eta_{ \pm}$in the helicity and $\sigma_{3}$ bases, defined in Eqs. (C7) and (C16), respectively, are related to each other via a linear transformation

$$
\begin{aligned}
\left(\begin{array}{c}
\eta_{+} \\
\eta_{-}
\end{array}\right) & =\left(\begin{array}{cc}
0 & 1 \\
-1 & 0
\end{array}\right)\left(\begin{array}{c}
\chi_{+}\left(\boldsymbol{n}_{\boldsymbol{p}}\right) \\
\chi_{-}\left(\boldsymbol{n}_{\boldsymbol{p}}\right)
\end{array}\right) \\
& =\left(\begin{array}{cc}
\sin \frac{\theta}{2} & \cos \frac{\theta}{2} e^{i \varphi} \\
-\cos \frac{\theta}{2} e^{-i \varphi} & \sin \frac{\theta}{2}
\end{array}\right)\left(\begin{array}{c}
\chi_{+}\left(\boldsymbol{n}_{p^{\prime}}\right) \\
\chi_{-}\left(\boldsymbol{n}_{p^{\prime}}\right)
\end{array}\right) .
\end{aligned}
$$

Eq. (C36) allows one to relate the nucleon currents and scalar products $(l, h)$ calculated in the two bases

$$
\begin{aligned}
& \left(l, h_{++}^{\eta}\right)=\sin \frac{\theta}{2}\left(l, h_{+-}^{\chi}\right)+\cos \frac{\theta}{2} e^{-i \varphi}\left(l, h_{--}^{\chi}\right), \\
& \left(l, h_{-+}^{\eta}\right)=-\cos \frac{\theta}{2} e^{i \varphi}\left(l, h_{+-}^{\chi}\right)+\sin \frac{\theta}{2}\left(l, h_{--}^{\chi}\right)
\end{aligned}
$$

and

$$
\begin{aligned}
& \left(l, h_{+-}^{\eta}\right)=-\sin \frac{\theta}{2}\left(l, h_{++}^{\chi}\right)-\cos \frac{\theta}{2} e^{-i \varphi}\left(l, h_{-+}^{\chi}\right), \\
& \left(l, h_{--}^{\eta}\right)=\cos \frac{\theta}{2} e^{i \varphi}\left(l, h_{++}^{\chi}\right)-\sin \frac{\theta}{2}\left(l, h_{-+}^{\chi}\right) .
\end{aligned}
$$

(i) The nucleon currents, e.g., in the left-hand-side of the first line in Eq. (C37) correspond to the scatterings when the spin projection on the fixed axis (incoming neutrino direction) does not change $\left(h_{++}^{\eta}\right)$ and is flipped $\left(h_{-+}^{\eta}\right)$. These currents can be uniquely described by linear combinations of the current with negative helicity in both initial and final states $h_{-}^{\chi}$ and of the current in which the initially negative helicity is flipped $h_{+-}^{\chi}$, as illustrated in Fig. 10.

(ii) The following equalities hold true

$$
\begin{aligned}
& \left|\left(l, h_{++}^{\eta}\right)\right|^{2}+\left|\left(l, h_{-+}^{\eta}\right)\right|^{2}=\left|\left(l, h_{+-}^{\chi}\right)\right|^{2}+\left|\left(l, h_{--}^{\chi}\right)\right|^{2}, \\
& \left|\left(l, h_{+-}^{\eta}\right)\right|^{2}+\left|\left(l, h_{--}^{\eta}\right)\right|^{2}=\left|\left(l, h_{++}^{\chi}\right)\right|^{2}+\left|\left(l, h_{-+}^{\chi}\right)\right|^{2} .
\end{aligned}
$$

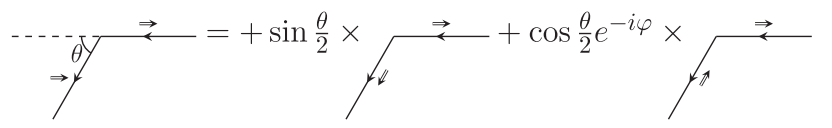

FIG. 10. The left pictogram corresponds to a fermion current $h_{++}^{\eta}$ with positive projections of its spin (shown by double arrowed line) on the given axis (shown by dashed line) in both initial and final states. This current is decomposed into a sum of the current with negative helicity in both the initial and final states $h_{-}^{\chi}$ and of the current in which the initially negative helicity is flipped $h_{+-}^{\chi}$ weighted with $\sin \frac{\theta}{2}$ and $\cos \frac{\theta}{2} e^{-i \varphi}$, respectively, as illustrated by the right pictogram.

(iii) Eqs. (C37) and (C38) can also be used to crosscheck the results of tedious calculations leading to Eq. (C17).

\section{APPENDIX D: AN ANALOGY IN A MECHANICAL SYSTEM FOR ELASCTIC AND INELASTIC SCATTERING}

Kinematic issues discussed in Sec. II C are valid not only for a quantum system but also for a mechanical system. As a useful illustration of elastic and inelastic scattering let us consider a system of two balls with equal masses $m$ connected by a massless spring with nonzero rigidity.

(i) Consider this system when both balls are at rest and one ball gains a momentum $\boldsymbol{q}$, and thus the kinetic energy $\boldsymbol{q}^{2} / 2 m$. After the acquired momentum is redistributed among the balls, their center-of-mass moves with momentum $\boldsymbol{q}$ but with a kinetic energy two time smaller, $\boldsymbol{q}^{2} / 4 m$, because of its mass $2 m$.

Half of the initial kinetic energy went into the potential energy of the spring. In this analogy, the initial ground state is transformed into an excited state. This example, shown in Fig. 11, illustrates an inelastic scattering.

(ii) Consider now the same balls in an initially excited state, i.e., moving towards and away from each other while their center-of-mass is at rest. The momenta of these balls at any time are equal to each other in magnitude, and have opposite directions. Let the

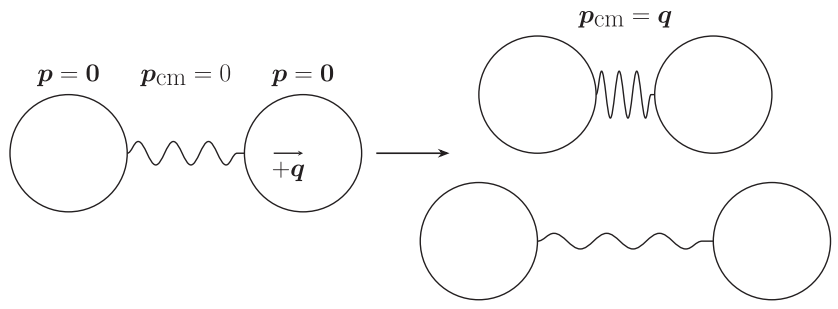

FIG. 11. Both balls are initially at rest when the right ball is hit with a momentum $\boldsymbol{q}$ (left). After some time their center-of-mass moves with momentum $\boldsymbol{q}$ and half of the transferred energy is accumulated in the potential energy of the spring, shown by its tension and extension (right). 


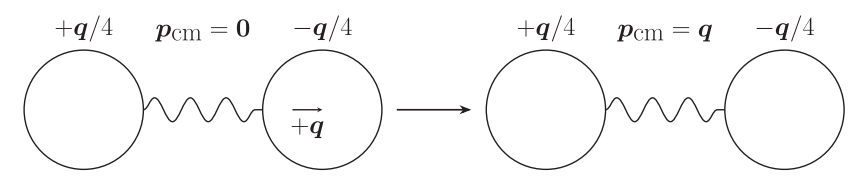

FIG. 12. Balls move towards and away each other with maximal momenta $\pm \boldsymbol{q} / 4$ while their center-of-mass is at rest, when the right ball having momentum $-\boldsymbol{q} / 4$ is hit and acquired the momentum $\boldsymbol{q}$ (left). After a while, their center-of-mass moves with momentum $\boldsymbol{q}$, while the spring still has the same potential energy, remaining in the same state. The balls have the same maximal momenta $\pm \boldsymbol{q} / 4$.

maximum momentum of a ball, reached when the spring has zero potential energy, be equal to $\pm \boldsymbol{q} / 4$ as shown in Fig. 12. One of these momenta is chosen in agreement with Eq. (31) to be $-\boldsymbol{q} / 4$. The total energy of both balls equals $(\boldsymbol{q} / 4)^{2} / 2 m+$ $(\boldsymbol{q} / 4)^{2} / 2 m=\boldsymbol{q}^{2} / 16 m$. Let the ball with a momentum $-\boldsymbol{q} / 4$ acquire an additional momentum $\boldsymbol{q}$.

Right after this interaction, the untouched and hit balls have momenta $(+\boldsymbol{q} / 4,+3 / 4 \boldsymbol{q})$ and energies $\left(\boldsymbol{q}^{2} / 32 m, 9 \boldsymbol{q}^{2} / 32 m\right)$, respectively. The total accumulated energy is equal to $5 q^{2} / 16 m$.

Since the kinetic energy of their center-of-mass equals $q^{2} / 4 m$, one finds that the potential energy accumulated in the spring is unchanged $\boldsymbol{q}^{2} / 16 \mathrm{~m}$.

In this example, the system of two balls connected by a spring remains in the state with the same internal potential energy. The change of kinetic energy of the struck ball is exactly the kinetic energy of their center-of-mass. This is an example of elastic scattering.
[1] D. Z. Freedman, Coherent neutrino nucleus scattering as a probe of the weak neutral current, Phys. Rev. D 9, 1389 (1974).

[2] A. Drukier and L. Stodolsky, Principles and applications of a neutral current detector for neutrino physics and astronomy, Phys. Rev. D 30, 2295 (1984).

[3] J. Barranco, O. G. Miranda, and T. I. Rashba, Probing new physics with coherent neutrino scattering off nuclei, J. High Energy Phys. 12 (2005) 021.

[4] K. Patton, J. Engel, G. C. McLaughlin, and N. Schunck, Neutrino-nucleus coherent scattering as a probe of neutron density distributions, Phys. Rev. C 86, 024612 (2012).

[5] D. K. Papoulias and T. S. Kosmas, Standard and Nonstandard Neutrino-Nucleus Reactions Cross Sections and Event Rates to Neutrino Detection Experiments, Adv. High Energy Phys. 2015, 763648 (2015).

[6] K. S. Kumar, S. Mantry, W. J. Marciano, and P. A. Souder, Low Energy Measurements of the Weak Mixing Angle, Annu. Rev. Nucl. Part. Sci. 63, 237 (2013).

[7] P. F. Smith, Coherent neutrino scattering - relativistic and nonrelativistic, Nuovo Cimento Soc. Ital Fis. A 83, 263 (1984).

[8] N. Jachowicz, K. Heyde, and S. Rombouts, Many-body description of neutrino-nucleus interactions, Nucl. Phys. A688, 593 (2001).

[9] P. C. Divari, V. C. Chasioti, and T. S. Kosmas, Neutral current neutrino- ${ }^{98} \mathrm{Mo}$ reaction cross sections at low and intermediate energies, Phys. Scr. 82, 065201 (2010).

[10] G. McLaughlin, Theory and phenomenology of coherent neutrino-nucleus scattering, AIP Conf. Proc. 1666, 160001 (2015).

[11] J. Engel, Nuclear form-factors for the scattering of weakly interacting massive particles, Phys. Lett. B 264, 114 (1991).

[12] P. S. Amanik and G. C. McLaughlin, Nuclear neutron form factor from neutrino nucleus coherent elastic scattering, J. Phys. G 36, 015105 (2009).
[13] P. S. Amanik and G. C. McLaughlin, Neutron form factor from neutrino-nucleus coherent elastic scattering, arXiv:0707.4191.

[14] G. I. Lykasov and V. A. Bednyakov, Neutrino-nucleus interactions at low energies within Fermi-liquid theory, Phys. Rev. C 76, 014622 (2007).

[15] K. M. Patton, G. C. McLaughlin, and K. Scholberg, Prospects for using coherent elastic neutrino-nucleus scattering to measure the nuclear neutron form factor, Int. J. Mod. Phys. E 22, 1330013 (2013).

[16] J. R. Wilson, Coherent Neutrino Scattering and Stellar Collapse, Phys. Rev. Lett. 32, 849 (1974).

[17] D. Z. Freedman, D. N. Schramm, and D. L. Tubbs, The weak neutral current and its effects in stellar collapse, Annu. Rev. Nucl. Part. Sci. 27, 167 (1977).

[18] J. Bernabeu, Low-Energy Elastic Neutrino-Nucleon and Nuclear Scattering and Its Relevance for Supernovae (CERN, 1975).

[19] S. Rombouts and K. Heyde, Calculation of neutrino-nucleus scattering cross-sections relevant for supernova processes, Nucl. Phys. A621, 371C (1997).

[20] P. C. Divari, Coherent and incoherent neutral current scattering for supernova detection, Adv. High Energy Phys. 2012, 379460 (2012).

[21] P. C. Divari, S. Galanopoulos, and G. A. Souliotis, Coherent scattering of neutral-current neutrinos as a probe for supernova detection, J. Phys. G 39, 095204 (2012).

[22] K. Scholberg, Prospects for measuring coherent neutrinonucleus elastic scattering at a stopped-pion neutrino source, Phys. Rev. D 73, 033005 (2006).

[23] P. deNiverville, M. Pospelov, and A. Ritz, Light new physics in coherent neutrino-nucleus scattering experiments, Phys. Rev. D 92, 095005 (2015).

[24] I. Esteban, M. C. Gonzalez-Garcia, M. Maltoni, I. MartinezSoler, and J. Salvado, Updated constraints on non-standard interactions from global analysis of oscillation data, arXiv: 1805.04530 . 
[25] M. Abdullah, J. B. Dent, B. Dutta, G. L. Kane, S. Liao, and L. E. Strigari, Coherent elastic neutrino nucleus scattering as a probe of a $Z^{\prime}$ through kinetic and mass mixing effects, Phys. Rev. D 98, 015005 (2018).

[26] Y. Farzan, M. Lindner, W. Rodejohann, and X.-J. Xu, Probing neutrino coupling to a light scalar with coherent neutrino scattering, J. High Energy Phys. 05 (2018) 066.

[27] J. Billard, J. Johnston, and B. J. Kavanagh, Prospects for exploring new physics in coherent elastic neutrino-nucleus scattering, arXiv:1805.01798.

[28] P. B. Denton, Y. Farzan, and I. M. Shoemaker, A plan to rule out large non-standard neutrino interactions after COHERENT Data, J. High Energy Phys. 07 (2018) 037.

[29] S.-F. Ge and I. M. Shoemaker, Constraining photon portal dark matter with texono and coherent data, arXiv: 1710.10889.

[30] D. K. Papoulias and T. S. Kosmas, COHERENT constraints to conventional and exotic neutrino physics, Phys. Rev. D 97, 033003 (2018).

[31] B. C. Caas, E. A. Garcs, O. G. Miranda, and A. Parada, Future perspectives for a weak mixing angle measurement in coherent elastic neutrino nucleus scattering experiments, Phys. Lett. B 784, 159 (2018).

[32] J. Papavassiliou, J. Bernabeu, and M. Passera, Neutrinonuclear coherent scattering and the effective neutrino charge radius, Proc. Sci. HEP2005 (2006) 192.

[33] J. A. Formaggio, E. Figueroa-Feliciano, and A. J. Anderson, Sterile neutrinos, coherent scattering and oscillometry measurements with low-temperature bolometers, Phys. Rev. D 85, 013009 (2012).

[34] A. J. Anderson, J. M. Conrad, E. Figueroa-Feliciano, C. Ignarra, G. Karagiorgi, K. Scholberg, M. H. Shaevitz, and J. Spitz, Measuring active-to-sterile neutrino oscillations with neutral current coherent neutrino-nucleus scattering, Phys. Rev. D 86, 013004 (2012).

[35] M. Cadeddu, C. Giunti, Y. F. Li, and Y. Zhang, Average CsI Neutron Density Distribution from COHERENT Data, Phys. Rev. Lett. 120, 072501 (2018).

[36] Yu. V. Gaponov and V. N. Tikhonov, Elastic scattering of low-energy neutrinos by atomic systems, Yad. Fiz. 26, 594 (1977).

[37] L. M. Sehgal and M. Wanninger, Atomic Effects in Coherent Neutrino Scattering, Phys. Lett. B 171, 107 (1986).

[38] R. R. Lewis, Coherent Detector for Low-energy Neutrinos, Phys. Rev. D 21, 663 (1980).

[39] C. J. Horowitz, K. J. Coakley, and D. N. McKinsey, Supernova observation via neutrino - nucleus elastic scattering in the CLEAN detector, Phys. Rev. D 68, 023005 (2003).

[40] Y. Giomataris and J. D. Vergados, A network of neutral current spherical TPC's for dedicated supernova detection, Phys. Lett. B 634, 23 (2006).

[41] H. T. Wong, H.-B. Li, J. Li, Q. Yue, and Z.-Y. Zhou, Research program towards observation of neutrino-nucleus coherent scattering, J. Phys. Conf. Ser. 39, 266 (2006).

[42] J. D. Vergados, F. T. Avignone, III, and I. Giomataris, Coherent neutral current neutrino-nucleus scattering at a spallation source: A Valuable Experimental Probe, Phys. Rev. D 79, 113001 (2009).
[43] S. Sangiorgio, A. Bernstein, J. Coleman, M. Foxe, C. Hagmann, T. H. Joshi, I. Jovanovic, K. Kazkaz, K. Movrokoridis, and S. Pereverzev, R\&D for the observation of coherent neutrino-nucleus scatter at a nuclear reactor with a dual-phase argon ionization detector, Phys. Procedia 37, 1266 (2012).

[44] S. J. Brice et al., A method for measuring coherent elastic neutrino-nucleus scattering at a far off-axis highenergy neutrino beam target, Phys. Rev. D 89, 072004 (2014).

[45] A. V. Kopylov, I. V. Orekhov, V. V. Petukhov, and A. E. Solomatin, Large gaseous detector of ionizing eradiation in search for coherent neutrino-nucleus scattering, arXiv: 1311.6564.

[46] A. V. Kopylov, I. V. Orekhov, V. V. Petukhov, and A. E. Solomatin, Gaseous detector of ionizing radiation for registration of coherent neutrino scattering on nuclei, Tech. Phys. Lett. 40, 185 (2014).

[47] G. Agnolet et al. (MINER Collaboration), Background studies for the MINER coherent neutrino scattering reactor experiment, Nucl. Instrum. Methods Phys. Res., Sect. A 853, 53 (2017).

[48] A. Aguilar-Arevalo et al. (CONNIE Collaboration), Proceedings, 15th Mexican Workshop on Particles and Fields (MWPF 2015): Mazatln, Mxico, 2015 [J. Phys. Conf. Ser. 761, 012057 (2016)].

[49] G. Fernandez Moroni, J. Estrada, E. E. Paolini, G. Cancelo, J. Tiffenberg, and J. Molina, Charge Coupled Devices for detection of coherent neutrino-nucleus scattering, Phys. Rev. D 91, 072001 (2015).

[50] V. Belov et al., The vGeN experiment at the Kalinin Nuclear Power Plant, J. Instrum. 10, P12011 (2015).

[51] R. Tayloe (COHERENT Collaboration), The CENNS-10 liquid argon detector to measure CE $\nu$ NS at the Spallation Neutron Source, J. Instrum. 13, C04005 (2018).

[52] J. Billard et al., Coherent neutrino scattering with low temperature bolometers at chooz reactor complex, J. Phys. G 44, 105101 (2017).

[53] H. T. Wong, Neutrino-nucleus coherent scattering and dark matter searches with sub-keV germanium detector, Nucl. Phys. A844, 229C (2010).

[54] A. J. Anderson, J. M. Conrad, E. Figueroa-Feliciano, K. Scholberg, and J. Spitz, Coherent neutrino scattering in dark matter detectors, Phys. Rev. D 84, 013008 (2011).

[55] A. Gutlein et al., Impact of coherent neutrino nucleus scattering on direct dark matter searches based on $\mathrm{CaWO}_{4}$ crystals, Astropart. Phys. 69, 44 (2015).

[56] V. A. Bednyakov, Is it possible to discover a dark matter particle with an accelerator?, Phys. Part. Nucl. 47, 711 (2016).

[57] S. Fallows, T. Kozynets, and C. B. Krauss, Sensitivity of the pico-500 bubble chamber to supernova neutrinos through coherent nuclear elastic scattering, arXiv:1806.01417.

[58] D. Aristizabal Sierra, V. De Romeri, and N. Rojas, COHERENT analysis of neutrino generalized interactions, arXiv:1806.07424.

[59] D. K. Papoulias, R. Sahu, T. S. Kosmas, V. K. B. Kota, and B. Nayak, Novel neutrino-floor and dark matter searches with deformed shell model calculations, Adv. High Energy Phys. 2018, 6031362 (2018). 
[60] A. Bolozdynya et al., Opportunities for neutrino physics at the spallation neutron source: A white paper, arXiv: 1211.5199.

[61] D. Akimov et al. (COHERENT Collaboration), The COHERENT experiment at the spallation neutron source, arXiv:1509.08702.

[62] J. I. Collar, N. E. Fields, M. Hai, T. W. Hossbach, J. L. Orrell, C. T. Overman, G. Perumpilly, and B. Scholz, Coherent neutrino-nucleus scattering detection with a CsI [Na] scintillator at the SNS spallation source, Nucl. Instrum. Methods Phys. Res., Sect. A 773, 56 (2015).

[63] D. Akimov et al. (COHERENT Collaboration), Observation of coherent elastic neutrino-nucleus scattering, Science 357, 1123 (2017).

[64] D. Akimov et al. (COHERENT Collaboration), COHERENT Collaboration data release from the first observation of coherent elastic neutrino-nucleus scattering, arXiv: 1804.09459.

[65] D. Akimov et al. (COHERENT Collaboration), COHERENT 2018 at the spallation neutron Source, arXiv: 1803.09183.

[66] I. Waller and D. R. Hartree, On the intensity of total scattering of x-rays, Proc. R. Soc. A 124, 119 (1929).

[67] P. M. Morse, Unelastische Streuung von Kathodenstrahlen, Phys. Z. 33, 443 (1932).

[68] L. Van Hove, Correlations in space and time and born approximation scattering in systems of interacting particles, Phys. Rev. 95, 249 (1954).

[69] S. Kerman, V. Sharma, M. Deniz, H. T. Wong, J. W. Chen, H. B. Li, S. T. Lin, C.-P. Liu, and Q. Yue (TEXONO Collaboration), Coherency in neutrino-nucleus elastic scattering, Phys. Rev. D 93, 113006 (2016).
[70] D. V. Naumov, Measurement of $\theta_{13}, \Delta m_{32}^{2}$ and covariant quantum-field theory of neutrino oscillations, D.Sc. dissertation, Dubna, JINR, 2017.

[71] D. V. Naumov and V. A. Naumov, A Diagrammatic treatment of neutrino oscillations, J. Phys. G 37, 105014 (2010).

[72] P. Pirinen, J. Suhonen, and E. Ydrefors, Neutral-current neutrino-nucleus scattering off $\mathrm{Xe}$ isotopes, arXiv: 1804.08995.

[73] V. Tsakstara, T. Kosmas, and J. Vergados, Weak responses of neutral current neutrino-nucleus reactions, Rom. J. Phys. 58, 1258 (2013).

[74] T. W. Donnelly and J. D. Walecka, Electron scattering and nuclear structure, Annu. Rev. Nucl. Part. Sci. 25, 329 (1975).

[75] D. Karlovets, Scattering of wave packets with phases, J. High Energy Phys. 03 (2017) 049.

[76] V. V. Belov et al., JINR Program Advisory Committee, 2018.

[77] D. Barker and D. M. Mei, Germanium detector response to nuclear recoils in searching for dark matter, Astropart. Phys. 38, 1 (2012).

[78] P. Agnes et al. (DarkSide Collaboration), Low-mass Dark Matter Search with the DarkSide-50 Experiment, arXiv: 1802.06994.

[79] J. Piekarewicz, A. R. Linero, P. Giuliani, and E. Chicken, Power of two: Assessing the impact of a second measurement of the weak-charge form factor of ${ }^{208} \mathrm{~Pb}$, Phys. Rev. C 94, 034316 (2016).

[80] R. H. Helm, Inelastic and elastic scattering of 187-Mev electrons from selected even-even nuclei,Phys. Rev. 104, 1466 (1956).

[81] D. V. Naumov, A jupyter notebook for neutrino-nucleus elastic scattering, https://git.jinr.ru/dnaumov/NNE (2018). 